\title{
Quantum mechanics in an evolving Hilbert space
}

\author{
Emilio Artacho 1,2,3 and David D. O’'Regan ${ }^{4,3}$ \\ ${ }^{1}$ CIC Nanogune and DIPC, Tolosa Hiribidea 76, 20018 San Sebastian, Spain \\ ${ }^{2}$ Ikerbasque, Basque Foundation for Science, 48011 Bilbao, Spain \\ ${ }^{3}$ Theory of Condensed Matter, Cavendish Laboratory, University of Cambridge, J. J. Thomson Avenue, Cambridge CB3 OHE, United Kingdom \\ ${ }^{4}$ School of Physics, CRANN, and AMBER, Trinity College Dublin, Dublin 2, Ireland
}

(Received 17 August 2016; revised manuscript received 8 January 2017; published 31 March 2017)

\begin{abstract}
Many basis sets for electronic structure calculations evolve with varying external parameters, such as moving atoms in dynamic simulations, giving rise to extra derivative terms in the dynamical equations. Here we revisit these derivatives in the context of differential geometry, thereby obtaining a more transparent formalization, and a geometrical perspective for better understanding the resulting equations. The effect of the evolution of the basis set within the spanned Hilbert space separates explicitly from the effect of the turning of the space itself when moving in parameter space, as the tangent space turns when moving in a curved space. New insights are obtained using familiar concepts in that context such as the Riemann curvature. The differential geometry is not strictly that for curved spaces as in general relativity, a more adequate mathematical framework being provided by fiber bundles. The language used here, however, will be restricted to tensors and basic quantum mechanics. The local gauge implied by a smoothly varying basis set readily connects with Berry's formalism for geometric phases. Generalized expressions for the Berry connection and curvature are obtained for a parameter-dependent occupied Hilbert space spanned by nonorthogonal Wannier functions. The formalism is applicable to basis sets made of atomic-like orbitals and also more adaptative moving basis functions (such as in methods using Wannier functions as intermediate or support bases), but should also apply to other situations in which nonorthogonal functions or related projectors should arise. The formalism is applied to the time-dependent quantum evolution of electrons for moving atoms. The geometric insights provided here allow us to propose new finite-difference time integrators, and also better understand those already proposed.
\end{abstract}

DOI: 10.1103/PhysRevB.95.115155

\section{INTRODUCTION}

Many electronic-structure methods use basis sets made of states that move or change with atomic positions. A very popular subset of these methods (most quantum-chemical [1-4] and a significant fraction of solid-state methods [5-9]) use atomic-like basis functions, composed of the product of a radial function and a spherical harmonic, normally centered around atoms. In other cases, the localized basis is obtained dynamically, using a finer auxiliary basis [10-12]. The detail of the kind of functions is not important in this work; what matters here is that such a basis is generally not orthonormal, it spans a subspace of the Hilbert space (a finite basis is always used), and both the basis and the subspace change with the evolution of a set of external parameters such as the atomic positions.

Nonorthogonal basis sets have been used since the early times of quantum mechanics, especially in the context of quantum chemistry [13]. The matrix representation of Schrödinger's equation (using Dirac notation) $H|\psi\rangle=E|\psi\rangle$ in a basis $\left\{\left|e_{\mu}\right\rangle, \mu=1, \ldots, \mathcal{N}\right\}$ gives

$$
\sum_{\nu} H_{\mu \nu} C_{\nu}=E \sum_{\nu} S_{\mu \nu} C_{\nu},
$$

where

$$
|\psi\rangle=\sum_{\mu}\left|e_{\mu}\right\rangle C_{\mu},
$$

$H_{\mu \nu}=\left\langle e_{\mu}|H| e_{\nu}\right\rangle$, and $S_{\mu \nu}=\left\langle e_{\mu} \mid e_{\nu}\right\rangle$, the latter being the overlap matrix.
Similarly, there are electronic structure methods based on the integration of time-evolving quantum problems, most prominently based on time-dependent density-functional theory [14-19]. The time-dependent Kohn-Sham equation arising is analogous to the time-dependent Schrödinger equation $H|\psi\rangle=i \partial_{t}|\psi\rangle$ (using $\hbar=m_{e}=e=1$ ), which, for a nonorthogonal basis set, becomes

$$
\sum_{\nu} H_{\mu \nu} C_{\nu}=i \sum_{\nu} S_{\mu \nu} \partial_{t} C_{\nu},
$$

in a situation in which the basis set is fixed [16]. If the basis set moves in time (e.g., related to nuclear motion) the equation becomes rather

$$
\sum_{\nu}\left(H_{\mu \nu}-i D_{\mu \nu}\right) C_{\nu}=i \sum_{\nu} S_{\mu \nu} \partial_{t} C_{\nu},
$$

in which the new terms $D_{\mu \nu}=\left\langle e_{\mu}\left|\partial_{t}\right| e_{\nu}\right\rangle=\left\langle e_{\mu} \mid \partial_{t} e_{\nu}\right\rangle$ appear related to the basis set evolution [20-22], although at sufficiently low nuclear velocities these terms can be neglected [23]. Similar objects to the $D_{\mu \nu}$ matrix in Eq. (2) can also be found in time-dependent methods using localized molecular orbitals [24]. Extra terms related to derivatives also appear when calculating the forces on atoms for geometry relaxation, $a b$ initio molecular dynamics calculations, or Ehrenfest dynamics simulations [20,21]. There are terms arising, called Pulay forces [25], which again involve basis vector derivatives.

The matrix representation of the quantum formalism used above has its limitations, however, and a more general formalization was introduced [26-29] based on tensors, which offers a better suited and more flexible framework for nonorthogonal basis sets, corresponding to a description of magnitudes in a 
Euclidean space with oblique axes. It allowed, for instance, the formalization of second quantization based on nonorthogonal bases [28], which was then used to formulate many-body theories using such bases [29,30] and to formulate corrective methods such as DFT $+U$ for the nonorthogonal case [31-33]. It also connected naturally with non-Hermitian representations proposed earlier for the better exploitation of localization [34-36].

In this paper we use concepts of differential geometry to extend that oblique-axis formalism to the calculation of derivatives when the basis and the Hilbert space it spans change with parameters such as atomic positions or time. This offers insights into the geometric interpretation of the dynamical equations arising with moving basis sets. In particular, the affine connection defined for the changing basis allows the proposal of optimized propagators for the numerical integration of quantum time-evolving problems. It should be noted that there have been previous works on derivatives in the tensorial formalism for nonorthogonal bases in electronic structure [37], including relaxations with curvy steps [38]. They were, however, always addressing derivatives of a scalar, the total energy, a special case which allows for circumvention of the key concepts in this work, the affine connection, and the covariant derivative. An alternative way of using differential geometry in electronic structure was initially explored in Ref. [39] when calculating derivatives with respect to the basis functions themselves, instead of external parameters as in this work. This is beyond the scope of the present paper.

The ideas in this paper should be useful for time-dependent (or parameter-dependent) methods involving basis functions, auxiliary support functions, or any kind of states, which move during simulations, including atomic-like basis orbitals, support functions, or generalized Wannier functions in largescale electronic-structure methods, or even the projectors for the core-electron description in projected-augmented-wave (PAW) methods. The connection is also made with the Berry formalism of geometric phases. In Sec. II the general formalism is presented, while Sec. III shows its application in several contexts. Many derivations have been pushed to appendices with a view to attaining a more concise exposition of the relevant ideas in the main text, while preserving a reasonably self-contained paper.

\section{FORMULATION}

\section{A. Tensorial representations}

In this work we will use tensorial representations as used in Ref. [28]. Here are the essentials before we get into derivatives. Consider a basis consisting on a set of linearly independent, nonorthogonal states,

$$
\left\{\left|e_{\mu}\right\rangle, \mu=1, \ldots, \mathcal{N}\right\},
$$

spanning a subspace $\Omega$ of the relevant Hilbert space $\mathcal{H}$ for our quantum problem. We will use here the tensorial notation for oblique angles [26-29]. For this, the dual basis [28,40] is defined as the set of vectors $\left\{\left|e^{\mu}\right\rangle, \mu=1, \ldots, \mathcal{N}\right\}$ in the same space $\Omega$ that fulfill

$$
\left\langle e^{\mu} \mid e_{v}\right\rangle=\delta_{\nu}^{\mu}=\left\langle e_{v} \mid e^{\mu}\right\rangle=\delta_{v}{ }^{\mu},
$$

where $\delta^{\mu}{ }_{v}=\delta_{v}{ }^{\mu}=\delta_{v}^{\mu}$ is Kronecker's delta. They also fulfill [28]

$$
\sum_{\mu}\left|e_{\mu}\right\rangle\left\langle e^{\mu}\left|=\sum_{\mu}\right| e^{\mu}\right\rangle\left\langle e_{\mu}\right|=P_{\Omega}
$$

where $P_{\Omega}$ is the projector onto the $\Omega$ Hilbert space. The metric tensors are given by the overlap, $S_{\mu \nu}=\left\langle e_{\mu} \mid e_{\nu}\right\rangle$, and its upperindices counterpart $S^{\mu \nu}=\left\langle e^{\mu} \mid e^{\nu}\right\rangle$, which is the inverse (in the matrix sense) of the overlap matrix.

In this paper we will mostly (always, unless explicitly stated) use the natural representation as defined in Ref. [28]. A state $|\psi\rangle \in \Omega$ is represented by the contravariant first-rank tensor

$$
\psi^{\mu}=\left\langle e^{\mu} \mid \psi\right\rangle,
$$

which corresponds to the coefficients $C_{\mu}$ of the vector expansion in Eq. (1), since

$$
|\psi\rangle=P_{\Omega}|\psi\rangle=\sum_{\mu}\left|e_{\mu}\right\rangle\left\langle e^{\mu} \mid \psi\right\rangle=\sum_{\mu}\left|e_{\mu}\right\rangle \psi^{\mu}=\left|e_{\mu}\right\rangle \psi^{\mu} .
$$

The last identity just reflects the fact that from now on we will use Einstein's convention for tensors [41], by which repeated indices imply a sum. A bra $\langle\psi| \in \Omega^{\dagger}$ will be represented by the equivalent covariant tensor,

$$
\psi_{\mu}=\left\langle\psi \mid e_{\mu}\right\rangle
$$

coming from

$$
\langle\psi|=\left\langle\psi \mid e_{\mu}\right\rangle\left\langle e^{\mu}\right|=\psi_{\mu}\left\langle e^{\mu}\right| .
$$

Note that the representation of the bra is not the complex conjugate of the representation of the ket (see Appendix A). The covariant and contravariant character of a tensor relates to the way they transform under basis change (see Appendix B for the definition).

An operator acting in $\Omega$ is represented by the second-rank tensor given by

$$
H_{v}^{\mu}=\left\langle e^{\mu}|H| e_{v}\right\rangle
$$

since

$$
P_{\Omega} H P_{\Omega}=\left(\left|e_{\mu}\right\rangle\left\langle e^{\mu}\right|\right) H\left(\left|e_{\nu}\right\rangle\left\langle e^{v}\right|\right)=\left|e_{\mu}\right\rangle H_{\nu}^{\mu}\left\langle e^{v}\right| .
$$

Schrödinger's equation $H|\psi\rangle=E|\psi\rangle$ in this representation then becomes

$$
H^{\mu}{ }_{\nu} \psi^{v}=E \psi^{\mu}
$$

It should be noted at this point that the formalism described here is equally valid for $|\psi\rangle$ being a single-particle or a many-particle state. It just requires the $\left|e_{\mu}\right\rangle$ basis states (and their duals) to represent the same number of particles as $|\psi\rangle$. One can also use this formalism for many-particle systems building on single-particle nonorthogonal basis states by using nonorthogonal second quantization [28,29]. In some parts below we will refer to single-particle (mean-field-like) situations since they are frequently found in different contexts, such as Kohn-Sham density-functional theory [42], but the present formalism is not limited to such situations.

The other representation to be considered in this work is the traditional quantum-chemical representation (henceforth called the matrix representation), which uses $\psi^{\mu}$ and 
$H_{\mu \nu}=\left\langle e_{\mu}|H| e_{\nu}\right\rangle$ for the representation of states and operators, respectively, the Schrödinger equation now reading

$$
H_{\mu \nu} \psi^{\nu}=E S_{\mu \nu} \psi^{\nu}
$$

as detailed in Ref. [28].

A Hermitian operator in the natural representation would fulfill

$$
H_{\nu}^{\mu}=\left(H_{v}^{\mu}\right)^{*}=\left(S_{\nu \lambda} H_{\sigma}^{\lambda} S^{\sigma \mu}\right)^{*},
$$

where $H_{v}{ }^{\mu}=\left\langle e_{v}|H| e^{\mu}\right\rangle$. In the matrix representation Hermiticity is reflected just by $H_{\mu \nu}=\left(H_{\nu \mu}\right)^{*}$. Further details on the tensorial notation used in this work are found in Appendix A.

All of these magnitudes are tensors in the sense that they represent abstract objects that are defined independently of the basis set. Tensor components transform in a well-defined fashion when changing the basis set. Transformations under basis change of the different tensors in this paper are discussed in Appendix B.

\section{B. Parameter vector space}

In this work we provide a comprehensive formalization of derivatives of the quantities just defined with respect to any parameters that the basis may depend on, including both the basis change within $\Omega$ and the evolution of $\Omega$ itself. Such parameters will normally be nuclear positions as in a molecular dynamics or Ehrenfest simulations, or time, as when following the dynamics governed by the time-dependent Schrödinger equation (or the time-dependent Kohn-Sham equation in timedependent density-functional theory, or analogous mean-fieldlike equation).

We will then consider those parameters as defining a vector space $\Theta$ of dimension $N$, spanned by the basis

$$
\left\{\mathbf{u}_{i}, i=1, \ldots, N\right\},
$$

such that any vector $\mathbf{R} \in \Theta$ is expanded as

$$
\mathbf{R}=R^{i} \mathbf{u}_{i} .
$$

Please keep in mind that these $R^{i}$ variables represent any parameters that a particular quantum problem may depend on, not necessarily nuclear positions (in the Applications section below there will be examples for nuclear positions, but also for time as a single parameter in a one-dimensional $\Theta$ ). We keep the tensor notation for the vectors in $\Theta$ for convenience. This allows for oblique angles in this space as well if ever wanted. We will always use Greek letters as indices for the quantum (electronic) components and Latin for the components of vectors in parameter space. Our electronic basis set and space do then depend on $\mathbf{R}$, i.e., $\Omega=\Omega(\mathbf{R}),\left|e_{\mu}\right\rangle=\left|e_{\mu}(\mathbf{R})\right\rangle$, and so will the projector $P_{\Omega}$ and all the tensors defined above.

It is important to note that, although we will exploit analogies with the differential geometry defined for curved spaces as in general relativity, the situation described here may be discussed more formally using the language of fiber bundles [43], with $\Theta$ as the base space and $\Omega(\mathbf{R})$ as a fiber for each $\mathbf{R}$. When moving in the base space, the Hilbert space associated with each fiber turns within the ambient Hilbert space $\mathcal{H}$, very much as the tangent space would turn for a curved space.
Although both the base space $\Theta$ and each fiber $\Omega(\mathbf{R})$ are each flat Euclidean spaces, the overall bundle is curved.

The parameter-dependent Hilbert space $\Omega(\mathbf{R})$ and its turning is also the basis of the Berry formalism of geometric phases in quantum mechanics $[44,45]$. In this case the relevant $\Omega$ space would be the one associated with the ground state, or, in a mean-field-like setting (as in, e.g., density-functional theory), the space spanned by the occupied single-particle states (occupied space), although also larger spaces are considered, e.g., for metallic systems, or disentangling bands [46]. We relate this work to the Berry formalism below. Finally, the change of basis between fibers can be regarded as a gauge transformation given in principle by any $\mathcal{N} \times \mathcal{N}$ invertible matrix of complex numbers, i.e., belonging to the general linear group $\operatorname{GL}(\mathcal{N}, \mathbb{C})$. In this paper, however, we prefer to introduce the formalism in an accessible, self-contained manner, using basic quantum mechanics, tensors, and simple manipulations therein.

In what follows, we will investigate the rate of change of quantum states, and operators acting upon them, with respect to the evolving basis vectors as we navigate the parameter vector space. We will find that the components of such change due to space preserving and space nonpreserving basis function evolution must be separately considered, as follows.

\section{Differential geometry}

\section{Covariant derivative}

The derivative of the $\psi^{\mu}$ components of a quantum state $|\psi\rangle$ with respect to $R^{i}$ will be indicated by

$$
\partial_{i} \psi^{\mu}=\frac{\partial \psi^{\mu}}{\partial R^{i}} .
$$

It is easy to show (see Appendix B) that such a derivative does not transform as a tensor under basis change. Using the conventional nomenclature: it is a nontensor.

Let us then define the covariant derivative as one that transforms as a tensor, which we can easily do as follows:

$$
ð_{i} \psi^{\mu} \equiv\left\langle e^{\mu}\right| P_{\Omega} \partial_{i}\left\{P_{\Omega}|\psi\rangle\right\}=\left\langle e^{\mu}\right| \partial_{i}\left\{P_{\Omega}|\psi\rangle\right\} .
$$

The projector directly acting on $|\psi\rangle$ might appear redundant if starting with $|\psi\rangle \in \Omega$. It is not so, however, the important point being that the derivative is calculated for the state being projected on the varying $\Omega$ space, and therefore, even though $|\psi\rangle$ and $P_{\Omega}|\psi\rangle$ are equal at $\mathbf{R}$, they are not necessarily equal at any nearby point, $\mathbf{R}+d R^{i} \mathbf{u}_{i}$. Put another way, the covariant derivative must be applied before projection onto $\Omega$, since both $|\psi\rangle$ and $P_{\Omega}$ may evolve in time.

This definition gives a well-behaved tensor since the defined $\widehat{\partial}_{i} \psi^{\mu}$ is the tensor representation of the vector $P_{\Omega} \partial_{i}\left\{P_{\Omega}|\psi\rangle\right\} \in$ $\Omega$ (see also Appendix B). The justification for this particular definition will become clear throughout this section. We can already point to the fact that for $|\psi\rangle \in \Omega$ such that neither $|\psi\rangle$ nor $\Omega$ change with $\mathbf{R}$, and for a basis set that does change, the proposed covariant derivative is zero (while the usual derivative is not), thereby indicating that it is the intrinsic rate of change of the state that is being measured, excluding the basis set change. This point will be proven more generally below. It is analogous to the definition of 
covariant derivatives in gauge-dependent theories, as the physical derivative independent of change of local gauge.

The relationship between $\partial_{i} \psi^{\mu}$ and $\partial_{i} \psi^{\mu}$ is obtained as follows:

$$
\begin{aligned}
\partial_{i} \psi^{\mu} & =\left\langle e^{\mu}\right| \partial_{i}\left\{P_{\Omega}|\psi\rangle\right\}=\left\langle e^{\mu}\right| \partial_{i}\left\{\left|e_{\nu}\right\rangle\left\langle e^{\nu} \mid \psi\right\rangle\right\} \\
& =\left\langle e^{\mu}\right| \partial_{i}\left\{\left|e_{\nu}\right\rangle \psi^{\nu}\right\}=\left\langle e^{\mu}\left|\partial_{i}\right| e_{\nu}\right\rangle \psi^{\nu}+\partial_{i} \psi^{\mu} .
\end{aligned}
$$

This gives us an alternative definition of the covariant derivative, expressed in objects all defined within $\Omega$, namely,

$$
ð_{i} \psi^{\mu}=\partial_{i} \psi^{\mu}+D_{\nu i}^{\mu} \psi^{\nu},
$$

where we have used the following definition:

$$
D_{\nu i}^{\mu} \equiv\left\langle e^{\mu}\left|\partial_{i}\right| e_{\nu}\right\rangle=\left\langle e^{\mu} \mid \partial_{i} e_{\nu}\right\rangle .
$$

The $i$ index is located after the index of the state being differentiated. This choice can be remembered by thinking of it as $\partial_{i}\left|e_{\mu}\right\rangle=\partial\left|e_{\mu}\right\rangle / \partial R^{i}$. This second expression of the covariant derivative [Eq. (4)] can be shown to give a tensor within this formalism (see Appendix B).

\section{Affine connection}

The quantity defined in Eq. (5) is also a nontensor and plays the role of the Christoffel symbols of the second kind in the Levi-Civita connection of conventional differential geometry. Keeping the nomenclature, our Christoffel symbols as defined in Eq. (5) thus define the affine connection relevant to our problem. Remember, however, that this is not differential geometry for curved spaces, where the tangent space at one point directly relates to the overall manifold, but rather for a rotating Hilbert space $\Omega$ within $\mathcal{H}$ when moving in parameter space $\Theta$. In the former, there is one metric tensor at every point, while in the latter there are still two metrics, one for $\Omega$ and one for $\Theta$. Hence, we do not establish a relationship between the defined Christoffel symbols and the electronic metric and its derivatives.

The defined Christoffel symbols exhibit other expected properties. They give, for instance, the expansion coefficients in $\Omega$ of the derivative of a basis vector:

$$
P_{\Omega} \partial_{i}\left|e_{\nu}\right\rangle=\left|e_{\mu}\right\rangle\left\langle e^{\mu}\left|\partial_{i}\right| e_{\nu}\right\rangle=\left|e_{\mu}\right\rangle D_{\nu i}^{\mu},
$$

so that when moving from one point $\mathbf{R} \in \Theta$ to another infinitesimally close to it $\mathbf{R}+d \mathbf{R}$, the basis vectors transform as

$$
\left|e_{\nu}(\mathbf{R}+d \mathbf{R})\right\rangle=\left|e_{\nu}(\mathbf{R})\right\rangle+\left|e_{\mu}(\mathbf{R})\right\rangle D_{\nu i}^{\mu} d R^{i},
$$

to linear order for infinitesimal $d \mathbf{R}$. The second term of the right-hand side accounts for the fact that not only the space is turning, but the basis set itself is changing when displacing in $\Theta$.

The turning of the Hilbert space $\Omega$ as we move in parameter space $\Theta$ demands the definition of the way the vector $|\psi(\mathbf{R})\rangle$ in $\Omega(\mathbf{R})$ propagates when moving to the neighboring point $\mathbf{R}+d \mathbf{R}$ into the slightly turned Hilbert space $\Omega(\mathbf{R}+d \mathbf{R})$. The required propagation is given by

$$
\psi^{\mu}(\mathbf{R}+d \mathbf{R})=\psi^{\mu}(\mathbf{R})+\partial_{i} \psi^{\mu}(\mathbf{R}) d R^{i},
$$

again for infinitesimal $d \mathbf{R}$. This means that the vector is first propagated in $\Omega(\mathbf{R})$ and then projected into $\Omega(\mathbf{R}+d \mathbf{R})$ by retaining the same vector components. This is consistent to linear order with the projective propagation of the state $|\psi\rangle$ when moving from $\mathbf{R}$ to $\mathbf{R}+d \mathbf{R}$,

$$
\begin{aligned}
|\psi(\mathbf{R}+d \mathbf{R})\rangle=P_{\Omega(\mathbf{R}+d \mathbf{R})} & \left\{P_{\Omega(\mathbf{R})}|\psi(\mathbf{R})\rangle\right. \\
+ & \left.P_{\Omega(\mathbf{R})} \partial_{i}\left[P_{\Omega(\mathbf{R})}|\psi(\mathbf{R})\rangle\right] d R^{i}\right\} .
\end{aligned}
$$

This last expression shows the propagation of a vector when moving in $\Theta$, irrespective of basis set and basis set transformation. It involves the covariant derivative in its final term. The equivalence between Eqs. (7) and (8) is shown in Appendix C.

Differently from canonical differential geometry of curved spaces, in the formalization presented here the proposed Christoffel symbols do not necessarily contract with the metric tensor following conventional rules for lowering or raising indices. The required equalities among these objects are presented in Appendix D.

\section{Covariant derivative for the bra representation}

The natural representation of the bra of any state, $\langle\psi|$, is $\psi_{\mu}=\left\langle\psi \mid e_{\mu}\right\rangle$. Its covariant derivative is defined in analogy to Eq. (3), namely,

$$
\mathrm{\partial}_{i} \psi \mu \equiv \partial_{i}\left\{\langle\psi| P_{\Omega}\right\}\left|e_{\mu}\right\rangle,
$$

which, again working in analogy with that which was done for Eq. (4), gives

$$
ð_{i} \psi_{\mu}=\partial_{i} \psi_{\mu}+\psi_{\nu} D_{i \mu}^{\nu},
$$

where we have defined the corresponding Christoffel symbol

$$
D_{i \mu}^{v} \equiv\left\langle\partial_{i} e^{v} \mid e_{\mu}\right\rangle
$$

[see the different order of indices in the symbol as compared to the definition in Eq. (5)].

The Christoffel symbols of Eqs. (5) and (11) are easy to interrelate, since $\left\langle e^{\mu} \mid e_{\nu}\right\rangle=\delta^{\mu}{ }_{\nu}$ for all $\mathbf{R} \in \Theta$, and, therefore,

$$
\partial_{i} \delta^{\mu}{ }_{\nu}=\left\langle\partial_{i} e^{\mu} \mid e_{\nu}\right\rangle+\left\langle e^{\mu} \mid \partial_{i} e_{\nu}\right\rangle=D_{i v}^{\mu}+D_{\nu i}^{\mu}=0,
$$

which leads to

$$
D_{i v}^{\mu}=-D_{\nu i}^{\mu}
$$

(general relations among Christoffel symbols can be found in Appendix D). The covariant derivative for the bra can then be written as

$$
ð_{i} \psi_{\mu}=\partial_{i} \psi_{\mu}-\psi_{\nu} D_{\mu i}^{\nu} .
$$

This derivative transforms as the corresponding tensor, as can be easily checked following any of the two procedures used above (and in Appendix B) for the case of the ket representation.

\section{Covariant derivative for operators}

Following the spirit of Eqs. (3) and (9), let us define the covariant derivative of an operator $H$ in its natural representation $H_{v}^{\mu}$ as

$$
ð_{i} H^{\mu} \equiv\left\langle e^{\mu}\left|\partial_{i}\left\{P_{\Omega} H P_{\Omega}\right\}\right| e_{v}\right\rangle,
$$

which becomes

$$
ð_{i} H_{\nu}^{\mu}=\partial_{i} H_{v}^{\mu}+D_{\lambda i}^{\mu} H_{v}^{\lambda}-H_{\lambda}^{\mu} D_{\nu i}^{\lambda} .
$$


For the last term we have used Eq. (12). This last expression coincides with the usual definition of the covariant derivative of a second-rank tensor in other differential geometry contexts, including general relativity, once the connection (the Christoffel symbols) has been defined. It can also be written as

$$
{\partial_{i}}_{H^{\mu}}^{\mu}=\partial_{i} H^{\mu}+[D, H]_{\nu i}^{\mu},
$$

where the commutator in the last term is defined as the two last terms in Eq. (15).

This definition gives a well-behaved tensor, transforming as such under a basis set change. It can be straightforwardly checked following either of the two procedures used before for $\mathrm{\partial}_{i} \psi^{\mu}$ : by noticing from Eq. (14) that it is a tensor representation of an operator within $\Omega$, and by following Appendix B. It is also a definition consistent with the previous ones. The following Leibniz chain rule for a vector,

$$
ð_{i}\left(H_{\nu}^{\mu} \psi^{v}\right)=\left(ð_{i} H_{\nu}^{\mu}\right) \psi^{v}+H_{\nu}^{\mu}\left(ð_{i} \psi^{v}\right),
$$

and the expected behavior of a scalar (zero-rank tensor),

$$
ð_{i} E=ð_{i}\left(\psi_{\mu} H^{\mu}{ }_{\nu} \psi^{\nu}\right)=\partial_{i}\left(\psi_{\mu} H_{\nu}^{\mu} \psi^{\nu}\right)=\partial_{i} E,
$$

are proved in Appendix E.

\section{Matrix representation}

Let us extend the previous definitions to the matrix representation, which will be useful in the next section. Since the ket is equally represented in the natural and matrix representations, the covariant derivative of a ket is also equally defined, as in Eq. (4). The bra is, however, different, since $\langle\psi|$ is represented by $\psi^{\mu *}=\left\langle\psi \mid e^{\mu}\right\rangle$ (see details of present notation in Appendix A). Its covariant derivative is then defined as

$$
\mathrm{\partial}_{i} \psi^{\mu *}=\partial_{i}\left\{\langle\psi| P_{\Omega}\right\}\left|e^{\mu}\right\rangle,
$$

and, therefore, without resorting to the ambient Hilbert space $\mathcal{H}$, it is expressed as

$$
\partial_{i} \psi^{\mu *}=\partial_{i} \psi^{\mu *}+\psi^{\nu *} D_{\nu i}^{\mu},
$$

which is just the Hermitian conjugate of Eq. (4) (see Appendix D for relations among Christoffel symbols). For an operator in this representation, the covariant derivative is defined as

$$
ð_{i} H_{\mu \nu}=\left\langle e_{\mu}\left|\partial_{i}\left\{P_{\Omega} H P_{\Omega}\right\}\right| e_{\nu}\right\rangle,
$$

which becomes

$$
ð_{i} H_{\mu \nu}=\partial_{i} H_{\mu \nu}+D_{\mu i}^{\sigma} H_{\sigma \nu}+H_{\mu \sigma} D_{i \nu}^{\sigma} .
$$

\section{Geometric interpretation of the affine connection}

Equations (7) and (8) give the basis for a clearer geometric interpretation of the affine connection defined above, and the corresponding covariant derivative. Closing Eq. (8) from the left with $\left\langle e^{\mu}(\mathbf{R}+d \mathbf{R})\right|$, one obtains

$$
\psi^{\mu}(\mathbf{R}+d \mathbf{R})=A^{\mu}{ }_{\nu}(\mathbf{R}+d \mathbf{R}: \mathbf{R})\left\{\psi^{v}+\left(ð_{i} \psi^{\nu}\right) d R^{i}\right\},
$$

where the terms within the curly brackets are defined at $\mathbf{R}$, and

$$
A^{\mu}{ }_{v}(\mathbf{R}+d \mathbf{R}: \mathbf{R}) \equiv\left\langle e^{\mu}(\mathbf{R}+d \mathbf{R}) \mid e_{v}(\mathbf{R})\right\rangle
$$

defines the basis transformation (as in Appendix B) when moving from $\mathbf{R}$ to $\mathbf{R}+d \mathbf{R}$ [the linear equivalence between
Eqs. (19) and (7) is shown in Appendix G]. Equation (20) represents the local gauge transformation. In principle any invertible matrix with a smooth behavior with respect to $\mathbf{R}$ is allowed.

In Eq. (19), the basis set transformation information is now carried by the $A$ tensor instead of the Christoffel symbols. The Christoffel symbols would reappear in Eq. (19) if replacing the covariant derivative by its definition in terms of the regular derivative, Eq. (4). That would indeed defeat the purpose of Eq. (19) [and would bring us back to Eq. (7), as shown in the mentioned Appendix G]. The usefulness of expressing the propagation as in Eq. (19) becomes evident when using it, for instance, for finite-difference time integrators for solving the time-dependent Schrödinger equation, as we will do in Sec. III A and Appendix J, where we will essentially replace $ð_{t} \psi^{\mu}$ by $-i H_{\nu}^{\mu} \psi^{\nu}$.

In addition to its utility for integrators, it is presented here because it conveys the geometric meaning of the affine connection quite clearly: the covariant derivative is the intrinsic one, independent of the basis set change, accounting for both the physical variation of the state and the turning of the Hilbert space $\Omega$, while the Christoffel symbols linearly account for the basis set transformation.

\section{Rotation versus deformation}

So far we have talked about basis change or transformation in general. We make here the distinction between pure rotations of the basis and what we will call basis deformation (in analogy with elasticity theory). They are defined as the ones for antiHermitian and Hermitian $D_{\mu v i}$, respectively. A small arbitrary transformation will thus have a rotation and a deformation component, that can be obtained from $\left(D_{\mu \nu i}-D_{v \mu i}^{*}\right) / 2$ and $\left(D_{\mu v i}+D_{v \mu i}^{*}\right) / 2$, respectively.

Appendix $\mathrm{H}$ shows how a small unitary transformation of the basis, defined as one that keeps constant overlap (and therefore corresponding to basis rotations in $\Omega$ ) has an associated anti-Hermitian $D_{\mu \nu i}$ tensor. This consideration will be relevant in the Applications section below, in the context of finite-difference integrators.

\section{Parallel transport and unitary propagation}

The analog of the parallel transport in curved spaces would be the propagation of a state vector $|\psi\rangle \in \Omega(\mathbf{R})$, which, in itself, would not vary (it would be constant if $\Omega=\mathcal{H}$ ) when moving in $\Theta$ away from point $\mathbf{R}$. Such intrinsic constancy is reflected by a null covariant derivative,

$$
\partial_{i} \psi^{\mu}=\partial_{i} \psi^{\mu}+D_{\nu i}^{\mu} \psi^{v}=0 .
$$

Therefore, parallel transport of any vector along any line in $\Theta$ is then obtained from Eq. (7), propagating

$$
\psi^{\mu}(\mathbf{R}+d \mathbf{R})=\psi^{\mu}(\mathbf{R})-D^{\mu}{ }_{\nu i}(\mathbf{R}) \psi^{v}(\mathbf{R}) d R^{i}
$$

(for infinitesimal $d \mathbf{R}$ ) along the corresponding line.

Vectors that are orthogonal to each other at a given point would propagate keeping their orthogonality. Appendix F shows that any unitary propagation (one such that the condition $ð_{i}\left\{\psi_{n \mu} \psi_{m}^{\mu}\right\}=0$ is preserved) maintains the orthogonality of propagated vectors. Parallel transport is a special case, since $ð_{i} \psi_{m}^{\nu}=ð_{i} \psi_{\mu n}=0$. 
More generally, a set of vectors in parallel transport keep their mutual scalar products (this can also be seen following the reasoning of Appendix F). This also applies to the two metric tensors, which are composed precisely of the scalar products of basis vectors. Since such scalar products are preserved under parallel transport, then

$$
ð_{i} S_{\mu \nu}=ð_{i} S^{\mu \nu}=0,
$$

which reflects a fundamental property of the theory, that the covariant derivative conserves the metric. This result is also derived explicitly in Appendix D.

\section{Curvature}

The Riemann-Christoffel curvature for a curved space characterizes the fact that if you take one vector in a vector field using the Levi-Civita connection along one direction, and then along another direction to reach a certain point, it gives a different result than if changing the order of directions in which you arrive to the same point (or analogously, if following a closed loop). This is locally quantified with the difference in changing the order of second derivatives, by defining the curvature tensor $R_{i v j}^{\mu}$ such that

$$
R_{i v j}^{\mu} \psi^{v}=ð_{i} ð_{j} \psi^{\mu}-ð_{j} ð_{i} \psi^{\mu} .
$$

Using the covariant derivatives defined above, one obtains

$$
R_{i v j}^{\mu}=\partial_{i} D_{\nu j}^{\mu}-\partial_{j} D_{\nu i}^{\mu}+D_{\lambda i}^{\mu} D_{\nu j}^{\lambda}-D_{\lambda j}^{\mu} D_{\nu i}^{\lambda},
$$

again in perfect analogy to the expression for curved spaces.

\section{Relation to Berry connection and curvature}

In Sec. II B the analogy with Berry's geometric phase formalism [45] was mentioned. Indeed, for a quantummechanical (single- or many-particle) state $|\Psi(\mathbf{R})\rangle$, the Berry connection is normally defined as

$$
\mathcal{A}_{j}=i\left\langle\Psi \mid \partial_{j} \Psi\right\rangle
$$

( $i=\sqrt{-1}$ ), which is nothing but ( $i$ times) the connection defined in this work [Eq. (5)] for a space $\Omega$ spanned by a single state. It generalizes to the trace

$$
\mathcal{A}_{j}=i \sum_{n}^{\mathrm{occ}}\left\langle\psi_{n} \mid \partial_{j} \psi_{n}\right\rangle,
$$

for a set of single-particle states $\left|\psi_{n}\right\rangle$ spanning the occupied space in the context of a mean-field-like approach to the manyparticle problem (as is the case for the Kohn-Sham states in density-functional theory). As expected from Berry's work, it is easy to see that

$$
\mathcal{A}_{j}=i D_{\mu j}^{\mu},
$$

where $D_{\mu j}^{\mu}$ is the trace of the connection defined in Eq. (5) (bear in mind that the $\left|\psi_{n}\right\rangle$ states in this context play the role of the basis of the relevant space, i.e., the $\left|e_{\mu}\right\rangle$ states of the previous sections, and $\Omega$ refers here to the occupied space). The expression in Eq. (23) assumes orthonormal states, whereas Eq. (24) is valid for any nonorthogonal basis of the relevant $\Omega$ space. More generally, the Berry connection matrix $\mathcal{A}_{m n j}=i\left\langle\psi_{m} \mid \partial_{j} \psi_{n}\right\rangle$ corresponds to ( $i$ times) this work's $D_{m n j}$ connection in the matrix representation, which can be transformed to any other tensorial representation for nonorthogonal states.

Similarly to what happens to the connection, the curvature of this work and that of Berry are closely related. The Berry curvature is usually defined as

$$
\mathcal{R}_{i j}=-2 \operatorname{Im}\left\{\left\langle\partial_{i} \Psi \mid \partial_{j} \Psi\right\rangle\right\},
$$

for a quantum mechanical state $|\Psi\rangle$, which generalizes to

$$
\mathcal{R}_{i j}=-2 \operatorname{Im}\left\{\sum_{n}^{\text {occ }}\left\langle\partial_{i} \psi_{n} \mid \partial_{j} \psi_{n}\right\rangle\right\}
$$

for a set of single-particle states $\left|\psi_{n}\right\rangle$ spanning the occupied space. The curvatures in Eqs. (22) and (25) are very closely interrelated when considering our $\Omega$ Hilbert space as the occupied space (or any specific subspace that we are computing the curvature for). Starting with

$$
\partial_{i} D_{\nu j}^{\mu}=\left\langle\partial_{i} e^{\mu} \mid \partial_{j} e_{\nu}\right\rangle-\left\langle e^{\mu} \mid \partial_{i} \partial_{j} e_{\nu}\right\rangle,
$$

we can easily see that

$$
\partial_{i} D_{\nu j}^{\mu}-\partial_{j} D_{\nu i}^{\mu}=\left\langle\partial_{i} e^{\mu} \mid \partial_{j} e_{\nu}\right\rangle-\left\langle\partial_{j} e^{\mu} \mid \partial_{i} e_{\nu}\right\rangle .
$$

If we now trace over the quantum variables, in analogy with the Ricci curvature

$$
\mathcal{R}_{i j}=R^{\mu}{ }_{i \mu j}=\left\langle\partial_{i} e^{\mu} \mid \partial_{j} e_{\mu}\right\rangle-\left\langle\partial_{j} e^{\mu} \mid \partial_{i} e_{\mu}\right\rangle,
$$

since the following traces annihilate:

$$
D_{\lambda i}^{\mu} D_{\mu j}^{\lambda}-D_{\lambda j}^{\mu} D_{\mu i}^{\lambda}=0 .
$$

If the basis $\left\{\left|e_{\mu}\right\rangle\right\}$ is invariably orthonormal, then $\left\langle e^{\mu}\right|=$ $\left\langle e_{\mu}\right|$, for any $\mathbf{R}$, and thus $\left\langle\partial_{i} e^{\mu}\right|=\left\langle\partial_{i} e_{\mu}\right|$, and

$$
\left\langle\partial_{j} e_{\mu} \mid \partial_{i} e_{\mu}\right\rangle=\left\langle\partial_{i} e_{\mu} \mid \partial_{j} e_{\mu}\right\rangle^{*},
$$

and, therefore,

$$
\mathcal{R}_{i j}=2 i \operatorname{Im}\left\{\left\langle\partial_{i} e_{\mu} \mid \partial_{j} e_{\mu}\right\rangle\right\},
$$

which is nothing but Eq. (25) (times $i$ ) for the $\left|\psi_{n}\right\rangle$ states taken as an orthonormal basis of occupied space $\Omega$. Therefore, Berry's curvature is nothing but the Ricci curvature of our turning occupied space.

This result is directly generalizable to any other orthonormal basis of occupied space, e.g., a basis of Wannier functions, still under Eq. (27). If the basis is nonorthogonal (nonorthogonal Wannier functions), Eq. (26) is then the relevant definition. If seeking an expression closer to Eq. (27), the definition in Eq. (26) can also be reexpressed as

$$
\begin{aligned}
\mathcal{R}_{i j}= & 2 i \operatorname{Im}\left\{S^{\mu \nu}\left\langle\partial_{i} e_{\nu} \mid \partial_{j} e_{\mu}\right\rangle\right\} \\
& +\left(\partial_{i} S^{\mu \nu}\right) D_{\nu \mu j}-\left(\partial_{j} S^{\mu \nu}\right) D_{\nu \mu i},
\end{aligned}
$$

which, in addition to the expected redefinition of the trace with the metric tensor in the first term, includes two additional terms related to the variation of the metric itself.

\section{The topology of $\Omega(\mathrm{R})$}

When solving a quantum-mechanical problem using a finite basis set that changes in parameter space, we will then have two relevant fiber bundles, one within the other: the one spanned by the basis, $\Omega$, and the one spanned by the occupied states. The curvature and topology of $\Omega(\mathbf{R})$ and its relation 
with the ones corresponding to the occupied space could have implications on the effect of the approximation implied by the basis. We will not explore this point further in this paper. We can, however, point to Ref. [47] for the implications of Berry concepts on the dependence of the occupied space on atomic positions in molecular systems, including the effect of conical intersections, for instance. Rethinking such ideas considering a wider-than-occupied space could be an avenue for future investigation.

\section{APPLICATIONS}

\section{A. Quantum time evolution}

\section{Basic equations}

Using the present formalism, we can consider a onedimensional parameter space with time as the only variable. In the natural representation, the time-dependent Schrödinger equation $H|\psi\rangle=i \partial_{t}|\psi\rangle$ becomes simply

$$
H_{\nu}^{\mu} \psi^{v}=i \partial_{t} \psi^{\mu},
$$

where the time covariant derivative is defined as

$$
\partial_{t} \psi^{\mu}=\partial_{t} \psi^{\mu}+D_{v t}^{\mu} \psi^{\nu},
$$

and the corresponding temporal Christoffel symbol as

$$
D_{v t}^{\mu}=\left\langle e^{\mu}\left|\partial_{t}\right| e_{\nu}\right\rangle=\left\langle e^{\mu} \mid \partial_{t} e_{\nu}\right\rangle .
$$

Equation (29) reflects the physics in a basis-set independent form, in the sense that the well-behaved tensors in the equation all transform as in Appendix B, and transmit the physics of the original Schrödinger equation for an evolving basis set and Hilbert space. Equation (29) can be obtained by minimizing the action for the following Lagrangian:

$$
L=i \psi_{\mu} ð_{t} \psi^{\mu}-\psi_{\mu} H_{\nu}^{\mu} \psi^{\nu},
$$

which is easily obtained using the ideas above from the standard $L=i\left\langle\psi \mid \partial_{t} \psi\right\rangle-\langle\psi|H| \psi\rangle$.

The matrix representation also allows a concise representation of the physical equation, albeit less elegantly, carrying around the metric tensors, as follows:

$$
H_{\mu \nu} \psi^{\nu}=i S_{\mu \nu} \partial_{t} \psi^{\nu},
$$

which corresponds to Eq. (2) of the Introduction, or

$$
S^{\mu \sigma} H_{\sigma \nu} \psi^{\nu}=i \partial_{t} \psi^{\mu} \text {. }
$$

If following the propagation of the density matrix instead of that of the wave functions, the dynamics is defined by the Liouville-von Neumann equation,

$$
i \partial_{t} \rho=[H, \rho],
$$

where the density operator $\rho$ would be

$$
\rho(t)=|\Psi(t)\rangle\langle\Psi(t)|
$$

for a pure quantum state, or

$$
\rho(t)=\sum_{n}^{\text {occ }}\left|\psi_{n}(t)\right\rangle\left\langle\psi_{n}(t)\right|
$$

for the set of occupied states in a mean-field setting. (It can also be generalized to statistical mixtures in general, including thermal.)
Again, for the evolving Hilbert space $\Omega$, the expression of this equation in terms of the corresponding tensors is obtained by closing it with $\left\langle e^{\mu}\right|$ from the left and $\left|e_{\nu}\right\rangle$ from the right, and substituting $\rho$ and $H$ by $P_{\Omega} \rho P_{\Omega}$ and $P_{\Omega} H P_{\Omega}$, giving

$$
i{ }_{\partial_{t}} \rho^{\mu}{ }_{\nu}=[H, \rho]^{\mu}{ }_{v}=H^{\mu}{ }_{\sigma} \rho^{\sigma}{ }_{v}-\rho^{\mu}{ }_{\sigma} H^{\sigma}{ }_{v}
$$

in its natural representation. The matrix representation of this equation is much less elegant. The conventional definition of the density matrix in a typical quantum chemistry setting is $\sum_{n}^{\mathrm{occ}} C_{\mu n} C_{v n}^{*}$, in the language of Eq. (1) of the Introduction. This is nothing but

$$
\rho^{\mu \nu}=\left\langle e^{\mu}|\rho| e^{v}\right\rangle .
$$

In this representation, the Liouville-von Neumann equation becomes

$$
i ð_{t} \rho^{\mu \nu}=S^{\mu \sigma} H_{\sigma \kappa} \rho^{\kappa \nu}-\rho^{\mu \sigma} H_{\sigma \kappa} S^{\kappa \nu} .
$$

For any of the former equations, the time dependence of the basis orbitals may be due to the variation in time of other parameters like atomic positions $R^{i}$. In such cases, the Christoffel symbols in the covariant derivatives would satisfy

$$
D_{\nu t}^{\mu}=v^{i} D_{\nu i}^{\mu}
$$

(or equivalent representations) where $v^{i}=\partial R^{i} / \partial t$ are the corresponding nuclear velocities.

\section{Crank-Nicholson integrator}

In various contexts a time-dependent Schrödinger-like equation is numerically solved by discretizing time, using adequate integrator algorithms (for a comparison of the performance and stability of different options see Refs. [48,49]). In the context of nonorthogonal basis sets and within meanfield-like theories for which matrix inversion is affordable, the Crank-Nicholson algorithm has been used quite successfully [16]. The generalization of that procedure to a moving basis set was achieved by incorporating a Löwdin orthonormalization step, an idea due to Sankey and collaborators [50], which will be discussed below. The Crank-Nicholson-Löwdin procedure proved quite successful in the integration of the Kohn-Sham equations for several studies of electronic stopping power for ionic projectiles shooting through varied materials [51-53]. Here we define new integrators based on the Crank-Nicholson idea, inspired by the affine connection defined above, and we compare them with the Crank-Nicholson-Löwdin procedure.

Let us first revise the Crank-Nicholson method in this context for nonmoving bases [16]. The basics: a state $|\psi\rangle$ evolving according to $H|\psi\rangle=i \partial_{t}|\psi\rangle$ can be propagated from $t$ to $t+d t$ by considering the backwards and forwards evolution from each of those time points to the one in the middle, as follows:

$$
\begin{aligned}
& |\psi(t+d t / 2)\rangle=\left\{1-i \frac{d t}{2} H(t)\right\}|\psi(t)\rangle, \\
& |\psi(t+d t / 2)\rangle=\left\{1+i \frac{d t}{2} H(t+d t)\right\}|\psi(t+d t)\rangle,
\end{aligned}
$$

which, by eliminating the middle point, becomes

$$
|\psi(t+d t)\rangle=\left[1+i \frac{d t}{2} H(t+d t)\right]^{-1}\left\{1-i \frac{d t}{2} H(t)\right\}|\psi(t)\rangle,
$$


thereby ensuring, by construction, that the algorithm respects invariance under time reversal.

The use of this algorithm is complicated by the dependence on $H(t+d t)$, which requires the use of an iterative selfconsistency procedure [23]. For practical purposes, however, in many implementations the algorithm is simplified by using $H(t)$ in both factors,

$$
|\psi(t+d t)\rangle=\left[1+i \frac{d t}{2} H(t)\right]^{-1}\left\{1-i \frac{d t}{2} H(t)\right\}|\psi(t)\rangle,
$$

which allows a direct evaluation of $|\psi(t+d t)\rangle$ from information of the previous time step. This change is of course of no consequence if the Hamiltonian does not change with time, but this is hardly the case for any mean-field-like Hamiltonian (such as that of Kohn and Sham), given the dependence of the Hamiltonian on the evolving electron density or wave functions.

For a fixed, not evolving basis set, this is used [16] as

$$
\begin{aligned}
\psi^{\mu}(t+d t)= & {\left[\delta_{\mu}{ }^{\sigma}+i \frac{d t}{2} H_{\mu}{ }^{\sigma}(t)\right]^{-1} } \\
& \times\left\{\delta^{\sigma}{ }_{v}-i \frac{d t}{2} H_{\nu}^{\sigma}(t)\right\} \psi^{v}(t)
\end{aligned}
$$

[please note that the first factor does not indicate an inversion of the particular $(\mu, \sigma)$ element, but of the tensor as a whole; see Appendix I for the notation and relevant definitions used in this paper for inverse second-rank tensors]. In the matrix representation it becomes

$$
\begin{aligned}
\psi^{\mu}(t+d t)= & {\left[S_{\mu \sigma}+i \frac{d t}{2} H_{\mu \sigma}(t)\right]^{-1} } \\
& \times\left\{S_{\sigma \nu}-i \frac{d t}{2} H_{\sigma \nu}(t)\right\} \psi^{\nu}(t),
\end{aligned}
$$

keeping in mind that the lower indices within the inverted tensor become upper indices (see Appendix I).

For fixed bases this algorithm has the enormous virtue of being strictly unitary [16], in the sense that when propagating an orthonormal set of states $\psi_{m}^{\mu}$, the set remains orthonormal at $t+d t$ regardless of the size of $d t$.

\section{Revising Crank-Nicholson for a moving basis}

For a moving basis set, the algorithm has to be generalized. One straightforward generalization is achieved by replacing $H_{\mu \sigma}(t)$ in Eq. (34) by $H_{\mu \sigma}(t)-i D_{\mu \sigma t}(t)$ (in the matrix representation, for instance), giving

$$
\begin{aligned}
\psi^{\mu}(t+d t)= & {\left[S_{\mu \sigma}+i \frac{d t}{2}\left(H_{\mu \sigma}-i D_{\mu \sigma t}\right)\right]^{-1} } \\
& \times\left\{S_{\sigma \nu}-i \frac{d t}{2}\left(H_{\sigma \nu}-i D_{\sigma v t}\right)\right\} \psi^{v}(t)
\end{aligned}
$$

(where we have dropped the $t$ dependence of the tensors for clarity). Here, $D_{\mu \sigma t}=\left\langle e_{\mu} \mid \partial_{t} e_{\sigma}\right\rangle$ is the required temporal Christoffel symbol. Equation (35) can be derived from Eq. (7) using the tensorial time-dependent Schrödinger equation in Eq. (29) and the definition of the covariant derivative, Eq. (4).
This would be only linearly correct in $d t$ in what concerns both Hilbert space turning and basis set transformation, and therefore the nice unitary propagation feature for arbitrary $d t$ is lost. Indeed, the loss of Hermiticity of the propagated effective Hamiltonian makes this problem apparent, albeit that the propagation is correct and well behaved in the limit of small $d t$.

A much more promising approach is obtained by building on Eq. (19) instead of Eq. (7), which exactly accounts for basis set change if $\Omega(t+d t)=\Omega(t)$. Within the matrix representation, one obtains

$$
\begin{aligned}
\psi^{\mu}(t+d t)= & {\left[S_{\mu \lambda}(t+d t)+i \frac{d t}{2} H_{\mu \lambda}(t+d t)\right]^{-1} } \\
& \times A_{\lambda}^{\sigma}(t+d t: t)\left\{S_{\sigma \nu}(t)-i \frac{d t}{2} H_{\sigma \nu}(t)\right\} \psi^{\nu}(t),
\end{aligned}
$$

where $A_{\lambda}^{\sigma}(t+d t: t)=\left\langle e_{\lambda}(t+d t) \mid e^{\sigma}(t)\right\rangle$ is defined analogously to Eq. (20). The derivation of Eq. (36) is given in Appendix J. The algorithm given by Eq. (36) would again require an iterative self-consistency procedure for every time step. An analog to Eq. (32) can also be considered with a view to removing the dependence on $H_{\mu \nu}(t+d t)$ and $S_{\mu \nu}(t+d t)$. This is achieved with the following Ansatz:

$$
\begin{aligned}
\psi^{\mu}(t+d t)= & A_{\lambda}^{\mu}(t+d t: t)\left[S_{\lambda \sigma}(t)+i \frac{d t}{2} H_{\lambda \sigma}(t)\right]^{-1} \\
& \times\left\{S_{\sigma \nu}(t)-i \frac{d t}{2} H_{\sigma \nu}(t)\right\} \psi^{\nu}(t) .
\end{aligned}
$$

The integrator in Eq. (37) keeps the strict unitary character of the algorithm for a transforming basis set in a fixed $\Omega$ space. This can be seen by noticing that the last two transformations in Eq. (37) (the ones in curly and square brackets) correspond to the original Crank-Nicholson scheme for a fixed Hamiltonian, and that the $A$ tensor transformation is nothing but a change of basis. The latter, however, is only linearly correct in $d t$ if $\Omega$ does turn. Indeed, for an arbitrarily large $d t$, the set $P_{\Omega(t+d t)}\left|\psi_{m}\right\rangle$ is not necessarily orthonormal even if the set $P_{\Omega(t)}\left|\psi_{m}\right\rangle$ was. The advantage of Eq. (37) with respect to Eq. (35) is important, however, since the space turning diminishes with better converged bases, while the basis change does not necessarily diminish (think of the perfectly converged limit of $\Omega=\mathcal{H}$ for a moving basis: the basis still changes and the Hilbert space does not).

In practice, the tensor $A_{\lambda}^{\mu}(t+d t: t)$ of Eq. (37) is somewhat inconvenient for calculations. It can be replaced by

$$
\begin{aligned}
\psi^{\mu}(t+d t)= & S^{\mu \kappa}(t+d t) A_{\kappa \lambda}(t+d t: t) \\
& \times\left[S_{\lambda \sigma}(t)+i \frac{d t}{2} H_{\lambda \sigma}(t)\right]^{-1} \\
& \times\left\{S_{\sigma \nu}(t)-i \frac{d t}{2} H_{\sigma \nu}(t)\right\} \psi^{\nu}(t),
\end{aligned}
$$

which involves the inversion of the overlap matrix at $t+d t$ and the calculation of the overlap between the $t$ basis vectors and the ones at $t+d t$ (in addition to the Crank-Nicholson 
operations at $t$ ). Similarly, the practical implementation of the self-consistent procedure implied by Eq. (36) would actually imply using the following self-consistent integrator instead:

$$
\begin{aligned}
\psi^{\mu}(t+d t)= & {\left[S_{\mu \lambda}(t+d t)+i \frac{d t}{2} H_{\mu \lambda}(t+d t)\right]^{-1} } \\
& \times A_{\lambda \kappa}(t+d t: t) \\
& \times\left\{\delta^{\kappa}{ }_{\nu}-i \frac{d t}{2} S^{\kappa \sigma}(t) H_{\sigma \nu}(t)\right\} \psi^{\nu}(t) .
\end{aligned}
$$

\section{Procedure based on Löwdin orthonormalization}

An alternative integrator was proposed [50] and has been used [51-53] for strict unitary propagation for arbitrary $d t$. Following the previous notation, this propagator can be written as

$$
\begin{aligned}
\psi^{\mu}(t+d t)= & \left\{S^{-1 / 2}(t+d t)\right\}^{\mu l}\left\{S^{1 / 2}(t)\right\}_{l \lambda} \\
& \times\left[S_{\lambda \sigma}(t)+i \frac{d t}{2} H_{\lambda \sigma}(t)\right]^{-1} \\
& \times\left\{S_{\sigma \nu}(t)-i \frac{d t}{2} H_{\sigma \nu}(t)\right\} \psi^{\nu}(t) .
\end{aligned}
$$

This is analogous to Eq. (38) inasmuch as it first does the physical (Crank-Nicholson) propagation for the basis at $t$ and within $\Omega(t)$, and then it transforms to conform to the basis at time $t+d t$. The basis transformation is done differently, however. It can be seen as the following process: (i) It first changes basis within $\Omega(t)$ to a Löwdin orthonormal basis. (ii) It then assumes that the coefficients in this basis do not change when changing to the Löwdin basis of $t+d t$. (iii) It then undoes the Löwdin transformation in $t+d t$ obtaining the sought coefficients in the nonorthogonal basis at $t+d t$.

This procedure has the advantage that the propagation is now strictly unitary by construction for any $d t$, even considering the turning of the Hilbert space, which should give larger stability to the method for relatively large values of $d t$. The propagated vectors are guaranteed to be orthonormal.

However, the vectors propagated following this Löwdin procedure [Eq. (40)] do not constitute a fair representation of what the evolution of the corresponding vectors would be in $\mathcal{H}$ in the sense that it does not properly counter the effect of the transforming basis. In order to see this, consider the case of a set of vectors at $t=0,\left\{\left|\psi_{n}\right\rangle\right\}$, all within $\Omega$ and all initially orthonormal, $\mathcal{S}_{n m}=\left\langle\psi_{n} \mid \psi_{m}\right\rangle=\delta_{n m}$. Consider, as well, that the dynamics is such that the vectors do not rotate with time, changing only in phase, as would be the case for eigenstates of a time-independent Hamiltonian, for instance. Take now a basis set for $\Omega$ that does rotate with time, but keeps orthonormality at all times. In this scenario it is clear that the coefficients $\psi_{n}^{\mu}$ should transform with time to capture the fact that the nonrotating eigenvectors are described in the frame of a rotating basis. This is properly taken care of in the integrator proposed in Eq. (38) by the $A_{\kappa \lambda}(t+d t: t)$ tensor, which does the needed basis transformation.

For the Löwdin procedure, however, the coefficients do not change, except for the global phase dictated by the Hamiltonian evolution, i.e.,

$$
\psi_{n}^{\mu}(t+d t)=e^{-i \omega_{n} d t} \psi_{n}^{\mu}(t)
$$

as is obvious from the fact that if $S_{\mu \nu}=\delta_{\mu \nu}$ at all times, then

$$
S_{\mu \nu}^{-1}=S^{-1 / 2 \mu \nu}=S_{\mu \nu}^{1 / 2}=\delta_{\mu \nu}
$$

at all times. This simple situation clearly illustrates the above assertion on the unfair representation by Eq. (38) of the states' evolution in a generic situation. It is not clear however how significant such discrepancies can be. In particular, the case made above is for pure rotations of the basis set. We explore this point in more depth in Appendix $\mathrm{K}$, finding interesting dependencies on the rotating versus deforming basis sets. In particular, for fixed-shape atomic-like orbitals as basis functions, such basis rotations correspond to Galileo transforms in parameter space, which would suggest no physical significance to the discrepancies between the solvers in Eqs. (40) and (38) for such rotations. This could be behind the apparent success of Eq. (40). A more quantitative analysis should be the focus of further work.

\section{Strictly unitary propagation}

If not using Eq. (40) for propagation, we are left with Eq. (38) [or Eq. (39) if using self-consistency], which does not strictly conserve the orthonormality of propagated states for finite $d t$ if the space $\Omega$ turns within $\mathcal{H}$. The propagator can still be used as long as $d t$ is small enough such that the unitary propagation is preserved within a desired tolerance. An alternative, however, is re-orthonormalizing the states. This can be done with any orthonormalization procedure, e.g., Gram-Schmidt or the iterative procedure used when finding eigenstates by minimization in electronic structure (see, e.g., Ref. [54]).

The Löwdin orthonormalization method described above can be used for this as well, with the advantage that the orthonormal states keep closest to the states prior to orthonormalization (we need to remember that we are not just after an orthonormal basis of the evolved occupied space, but actually following the evolution of separate states).

Consider a set of $M$ states, $\left\{\left|\psi_{n}\right\rangle, n=1, \ldots, M\right\}$, where $M<\mathcal{N}$, being $\mathcal{N}$ the dimension of $\Omega$, and which are represented by $\left\{\psi_{n}^{\mu}, n=1, \ldots, M ; \mu=1, \ldots, \mathcal{N}\right\}$, and are all, therefore, within $\Omega$. Consider they have been propagated by Eq. (38) and are not strictly orthonormal, i.e.,

$$
\mathcal{S}_{n m}=\left\langle\psi_{n} \mid \psi_{m}\right\rangle \neq \delta_{n m},
$$

where

$$
\mathcal{S}_{n m}=\psi_{\mu n} \psi_{m}^{\mu}
$$

is an $M \times M$ matrix. By diagonalizing $\mathcal{S}_{m n}$, one can obtain $\mathcal{S}^{-1 / 2 \mathrm{~nm}}$, whereupon the strictly unitary propagator version of Eq. (38) becomes

$$
\begin{aligned}
\psi_{n}^{\mu}(t+d t)= & \mathcal{S}^{-1 / 2 n m}(t+d t) S^{\mu \kappa}(t+d t) A_{\kappa \lambda}(t+d t: t) \\
& \times\left[S_{\lambda \sigma}(t)+i \frac{d t}{2} H_{\lambda \sigma}(t)\right]^{-1} \\
& \times\left\{S_{\sigma \nu}(t)-i \frac{d t}{2} H_{\sigma \nu}(t)\right\} \psi_{m}^{\nu}(t) .
\end{aligned}
$$


The self-consistent alternative orthonormalizing Eq. (39) would read

$$
\begin{aligned}
\psi_{n}^{\mu}(t+d t)= & \mathcal{S}^{-1 / 2{ }^{n m}}(t+d t) \\
& \times\left[S_{\mu \lambda}(t+d t)+i \frac{d t}{2} H_{\mu \lambda}(t+d t)\right]^{-1} \\
& \times A_{\lambda \kappa}(t+d t: t) \\
& \times\left\{\delta^{\kappa}{ }_{v}-i \frac{d t}{2} S^{\kappa \sigma}(t) H_{\sigma \nu}(t)\right\} \psi^{\nu}(t) .
\end{aligned}
$$

This orthonormalization step can be done at every evolution step, after a determined number of them, or when the deviation from orthonormality reaches some tolerance.

\section{B. Forces}

Additional terms related to derivatives also appear when calculating the forces on atoms for geometry relaxation or $a b$ initio molecular dynamics calculations. In the following we restrict the discussion to adiabatic forces, leaving for further work the consideration of additional terms that appear for moving basis sets in nonadiabatic settings [20]. Considering for simplicity one single state $|\psi\rangle$, and using the language of the Introduction, one calculates quantities like

$$
\begin{aligned}
\frac{\partial E}{\partial R_{i}}= & \partial_{i}\langle\psi|H| \psi\rangle=\partial_{i}\left\{\sum_{\mu \nu} C_{\mu}^{*}\left\langle e_{\mu}|H| e_{\nu}\right\rangle C_{\nu}\right\} \\
= & \sum_{\mu \nu}\left\{C_{\mu}^{*}\left\langle e_{\mu}\left|\partial_{i} H\right| e_{\nu}\right\rangle C_{\nu}\right. \\
& +\left(\partial_{i} C_{\mu}^{*}\right)\left\langle e_{\mu}|H| e_{\nu}\right\rangle C_{\nu}+C_{\mu}^{*}\left\langle e_{\mu}|H| e_{\nu}\right\rangle\left(\partial_{i} C_{\nu}\right) \\
& \left.+C_{\mu}^{*}\left\langle\partial_{i} e_{\mu}|H| e_{\nu}\right\rangle C_{\nu}+C_{\mu}^{*}\left\langle e_{\mu}|H| \partial_{i} e_{\nu}\right\rangle C_{\nu}\right\} .
\end{aligned}
$$

The last two terms, called Pulay forces [25], involve again basis vector derivatives. We discuss here the relevance of the present formalism for these forces and related concepts.

\section{Hellmann-Feynman theorem}

The Hellman-Feynman theorem states that, given $E=$ $\langle\psi|H| \psi\rangle$ (for a normalized $|\psi\rangle$ ), the derivative of $E$ with respect to $R^{i}$ fulfils

$$
\partial_{i} E=\left\langle\psi\left|\partial_{i} H\right| \psi\right\rangle
$$

if $H|\psi\rangle=E|\psi\rangle$ (and $\langle\psi| H=\langle\psi| E$ ). It is easy to see that, using the latter equations in $\Omega$,

$$
H_{\nu}^{\mu} \psi^{\nu}=E \psi^{\mu} \text { and } \psi_{\mu} H_{\nu}^{\mu}=\psi_{\nu} E,
$$

and the Hellman-Feynman theorem then reads

$$
\partial_{i} E=\psi_{\mu}\left(\partial_{i} H_{\nu}^{\mu}\right) \psi^{\nu} .
$$

In Eq. (17) we saw that the derivative of a scalar needs no correction. Actually,

$$
ð_{i} E=\partial_{i} E=\psi_{\mu}\left(\partial_{i} H_{\nu}^{\mu}\right) \psi^{v}=\psi_{\mu}\left(\partial_{i} H_{\nu}^{\mu}\right) \psi^{v},
$$

the last identity being easy to check by using Eqs. (15) and (45). This is a very transparent expression of the theorem in its general quantum mechanical form in Eq. (44).

\section{Hellman-Feynman theorem in matrix representation}

The matrix representation gives a less concise expression of the same theorem, except when using the covariant derivative of $H$. Starting with the Schrödinger equation, instead of Eq. (45), we have

$$
H_{\mu \nu} \psi^{\nu}=E S_{\mu \nu} \psi^{\nu} \text { and } \psi^{\mu *} H_{\mu \nu}=\psi^{\mu *} S_{\mu \nu} E .
$$

Expanding the derivative $\partial_{i} E=\partial_{i}\left(\psi^{\mu *} H_{\mu \nu} \psi^{\nu}\right)$ and using the fact that $\langle\psi \mid \psi\rangle=1=\psi^{\mu *} S_{\mu \nu} \psi^{\nu}$, the Hellman-Feynman theorem is obtained in this representation as

$$
\partial_{i} E=\psi^{\mu *}\left[\partial_{i} H_{\mu \nu}-E \partial_{i} S_{\mu \nu}\right] \psi^{\nu},
$$

which can also be expressed as

$$
\partial_{i} E=\psi^{\mu *}\left[\partial_{i} H_{\mu \nu}-E\left(D_{\mu i \nu}+D_{\mu \nu i}\right)\right] \psi^{\nu} .
$$

Let us see how it looks using the covariant derivative. Introducing its definition for the matrix representation of $H$ [Eq. (18)] into the previous expression [Eq. (48)],

$$
\begin{aligned}
\partial_{i} E= & \psi^{\mu *}\left[{\partial_{i}}_{i} H_{\mu \nu}-D_{\mu i}^{\sigma} H_{\sigma \nu}-H_{\mu \sigma} D_{i \nu}^{\sigma}\right. \\
& \left.-E\left(D_{\mu i \nu}+D_{\mu \nu i}\right)\right] \psi^{\nu} .
\end{aligned}
$$

Using now the Schrödinger equation again [Eq. (47)], the following elements of the previous equation become

$$
\begin{aligned}
& \psi^{\mu *} D_{\mu i}^{\sigma} H_{\sigma \nu} \psi^{\nu}=E \psi^{\mu *} D_{\mu i}{ }^{\sigma} S_{\sigma \nu} \psi^{\nu}, \\
& \psi^{\mu *} H_{\mu \sigma} D^{\sigma}{ }_{i \nu} \psi^{\nu}=E \psi^{\mu *} S_{\mu \sigma} D_{i \nu}^{\sigma} \psi^{\nu},
\end{aligned}
$$

whereupon Eq. (49) becomes

$$
\begin{aligned}
\partial_{i} E= & \psi^{\mu *}\left[\partial_{i} H_{\mu \nu}-E\left(D_{\mu i}{ }^{\sigma} S_{\sigma \nu}+S_{\mu \sigma} D_{i v}^{\sigma}\right.\right. \\
& \left.\left.+D_{\mu i v}+D_{\mu \nu i}\right)\right] \psi^{\nu} .
\end{aligned}
$$

Using now the relations between Christoffel symbols in Eq. (D2), we find that

$$
\begin{aligned}
& D_{\mu i}^{\sigma} S_{\sigma \nu}=-D_{\mu i}{ }^{\sigma} S_{\sigma \nu}=-D_{\mu i v}, \\
& S_{\mu \sigma} D_{i \nu}^{\sigma}=-S_{\mu \sigma} D^{\sigma}{ }_{\nu i}=-D_{\mu \nu i},
\end{aligned}
$$

and introducing these in Eq. (50) gives a quite simple final result for the Hellman-Feynman theorem in the matrix representation in terms of the Hamiltonian's covariant derivative, namely,

$$
\partial_{i} E=ð_{i} E=\psi^{\mu *}\left(ð_{i} H_{\mu \nu}\right) \psi^{\nu} .
$$

This last equation can also be derived directly from Eq. (46) using the fact that the covariant derivative conserves the metric, as discussed in Sec. II C 8, i.e., $ð_{i} S_{\mu \nu}=ð_{i} S^{\mu \nu}=0$. From Eq. (46), we have

$$
\begin{aligned}
& ð_{i} E=\psi_{\mu}\left(ð_{i} H_{\nu}^{\mu}\right) \psi^{\nu}=\psi^{\mu *} S_{\mu \lambda}\left(ð_{i} S^{\lambda \sigma} H_{\sigma \nu}\right) \psi^{\nu} \\
& =\psi^{\mu *} S_{\mu \lambda}\left\{S^{\lambda \sigma}\left(ð_{i} H_{\sigma \nu}\right)+\left(ð_{i} S^{\lambda \sigma}\right) H_{\sigma \nu}\right\} \psi^{\nu} \\
& =\psi^{\mu *} \delta_{\mu}^{\sigma}\left(ð_{i} H_{\sigma \nu}\right) \psi^{\nu}+\psi^{\mu *} S_{\mu \lambda} 0 H_{\sigma \nu} \psi^{\nu} \\
& =\psi^{\mu *}\left(\partial_{i} H_{\mu \nu}\right) \psi^{\nu},
\end{aligned}
$$

which is nothing but Eq. (51).

\section{Pulay forces}

When facing the problem of calculating the forces, $\partial_{i} E$, one still needs to calculate $\partial_{i} H_{\nu}^{\mu}$. For the time-dependent Schrödinger or the von Neumann equations above, the relevant 
derivatives were obtained by solving equations defined in (an evolving) $\Omega$. In this case, however, the calculation of $\partial_{i} H^{\mu}{ }_{v}$ is done by integration, effectively using an auxiliary basis set in $\mathcal{H}$ (analytically with Gaussians, on a real-space grid, etc.). The usual procedure follows

$$
\begin{aligned}
\partial_{i} H^{\mu} & =\partial_{i}\left\langle\psi^{\mu}|H| \psi_{\nu}\right\rangle \\
& =\left\langle e^{\mu}\left|\partial_{i} H\right| e_{\nu}\right\rangle+\left\langle\partial_{i} e^{\mu}|H| e_{\nu}\right\rangle+\left\langle e^{\mu}|H| \partial_{i} e_{\nu}\right\rangle .
\end{aligned}
$$

The last two terms give rise to the so-called Pulay terms [25], as already presented in Eq. (43). There is nothing substantially new offered by differential geometry in this context.

It is interesting, however, to separate the terms residing fully within $\Omega$ from the contributions outside it. Defining $Q_{\Omega}$ as $P_{\Omega}$ 's complement, i.e., $P_{\Omega}+Q_{\Omega}=\mathbb{1}$ (the identity operator in $\mathcal{H}$ ), we can expand

$$
\begin{aligned}
\left\langle\partial_{i} e^{\mu}|H| e_{\nu}\right\rangle & =\left\langle\partial_{i} e^{\mu}\left|\left(P_{\Omega}+Q_{\Omega}\right) H\right| e_{\nu}\right\rangle \\
& =\left\langle\partial_{i} e^{\mu} \mid e_{\sigma}\right\rangle\left\langle e^{\sigma}|H| e_{\nu}\right\rangle+\left\langle\partial_{i} e^{\mu}\left|Q_{\Omega} H\right| e_{\nu}\right\rangle \\
& =D^{\mu}{ }_{i \sigma} H^{\sigma}{ }_{\nu}+\left\langle\partial_{i} e^{\mu}\left|Q_{\Omega} H\right| e_{\nu}\right\rangle .
\end{aligned}
$$

Doing this for both Pulay terms and using the definition of the covariant derivative ${\partial_{i}}_{H^{\mu}}^{\mu}{ }_{\nu}$, one arrives at

$$
ð_{i} H^{\mu}{ }_{\nu}=\left\langle e^{\mu}\left|\partial_{i} H\right| e_{\nu}\right\rangle+\left\langle\partial_{i} e^{\mu}\left|Q_{\Omega} H\right| e_{\nu}\right\rangle+\left\langle e^{\mu}\left|H Q_{\Omega}\right| \partial_{i} e_{\nu}\right\rangle,
$$

the last two terms being explicitly built from the components out of $\Omega$ of both vectors $H\left|e_{\nu}\right\rangle$ and $\left|\partial_{i} e_{\nu}\right\rangle$ (and their duals/bras). Indeed, if neglecting out-of-space components, then

$$
\partial_{i} H_{\nu}^{\mu} \simeq\left\langle e^{\mu}\left|\partial_{i} H\right| e_{\nu}\right\rangle,
$$

and considering Eq. (46), we arrive at

$$
\partial_{i} E \simeq \psi_{\mu}\left\langle e^{\mu}\left|\partial_{i} H\right| e_{\nu}\right\rangle \psi^{\nu},
$$

or, in the matrix representation,

$$
\partial_{i} E \simeq \psi^{\mu *}\left\langle e_{\mu}\left|\partial_{i} H\right| e_{\nu}\right\rangle \psi^{\nu},
$$

where the Pulay terms have disappeared. Neglecting those terms, however, spoils the correspondence between $E$ and $\partial_{i} E$. This just reflects the fact that, in the time-dependent equations above, the derivatives of the basis vectors were naturally projected onto $\Omega$, which is not the case here.

\section{CONCLUSIONS}

Covariant derivatives are defined for derivatives of quantum-mechanical states in situations of basis sets varying as a function of external parameters. The concepts from differential geometry used to reformalize dynamical equations allow for better insights into the meaning of connection terms appearing in dynamical equations. In addition to relating to the Berry-phase and gauge formalisms, these geometrical insights enable the evaluation of existing, and proposal of new, finite-difference propagators for time evolution equations.

\section{ACKNOWLEDGMENTS}

We would like to thank Daniel Sanchez-Portal and Jorge Kohanoff for interesting and intense discussions on the problem of integrating time-dependent Kohn-Sham equations, Ivo Souza for discussions on the relation of this work with the Berry formalism, and Jonathan M. Evans for suggestions on the mathematics. D.D.O'R. would like to thank S. M.-M. Dubois, A. A. Mostofi, C.-K. Skylaris, and M. C. Payne for helpful comments an early stage of this work. Both authors would like to thank the anonymous reviewers for the careful reading of the manuscript and for their constructive comments, which have helped to improve the paper noticeably. E.A. acknowledges funding from the EU through the ElectronStopping Grant No. 333813, within the Marie-Curie CIG program, and from MINECO, Spain, through Grant No. FIS2015-64886-C5-1-P. D.D.O'R. gratefully acknowledges the support of the National University of Ireland and the School of Physics at Trinity College Dublin.

\section{APPENDIX A：TENSORIAL NOTATION}

The tensorial notation for this work presented in Sec. II A is different from the one in Ref. [28], and partly follows Ref. [29]. We do specify here an order for the tensor indices [29], which defines bra and ket components, instead of using barred indices as we did then [28]. The order of the indices matters as in $\left\langle e_{\mu}|H| e^{v}\right\rangle=H_{\mu}{ }^{\nu} \neq H_{\mu}^{\nu}$, and contractions are done on any two identical indices where one is up and the other is down, and where also one is left and the other is right. In order to differentiate between $\left\langle e_{\mu} \mid \psi\right\rangle$ and $\left\langle\psi \mid e_{\mu}\right\rangle$, and between $\left\langle e^{\mu} \mid \psi\right\rangle$ and $\left\langle\psi \mid e^{\mu}\right\rangle$, Ref. [29] used a dot to indicate order, as in $\psi_{\mu \bullet}=$ $\left\langle e_{\mu} \mid \psi\right\rangle$ and $\psi_{\bullet \mu}=\left\langle\psi \mid e_{\mu}\right\rangle$. We choose here to use only one index and explicit complex conjugation, with the convention that

$$
\begin{aligned}
\psi^{\mu} & =\left\langle e^{\mu} \mid \psi\right\rangle, & \psi_{\mu}=\left\langle\psi \mid e_{\mu}\right\rangle, \\
\psi^{\mu *} & =\left\langle\psi \mid e^{\mu}\right\rangle, & \psi_{\mu}^{*}=\left\langle e_{\mu} \mid \psi\right\rangle .
\end{aligned}
$$

With this choice the natural representation becomes very intuitive, with kets defined with upper index, bras with lower, and operators with upper for the bra (left) and lower for the ket (right). It is, however, less transparent for the raising or lowering of indices for these tensors. They carry an additional complex conjugation and one should keep in mind which ones are correct,

$$
\begin{aligned}
\psi_{\nu} S^{\nu \mu} & =\psi^{\mu *}, \quad S^{\mu \nu} \psi_{\nu}^{*}=\psi^{\mu}, \\
\psi^{\nu *} S_{\nu \mu} & =\psi_{\mu}, \quad S_{\mu \nu} \psi^{\nu}=\psi_{\mu}^{*},
\end{aligned}
$$

or, if preferred, go back to any of the other two options [28,29]. This is however not an issue for any of the manipulations found in this paper.

\section{APPENDIX B: TRANSFORMATION UNDER BASIS CHANGE}

We consider here the behavior under basis change of different tensors defined in this work. We first revise the expected behavior of a tensor before getting into derivatives. Take $\left\{\left|a_{n}\right\rangle\right\}$ as a new basis set of $\Omega$ that relates with the original $\left\{\left|e_{\mu}\right\rangle\right\}$ by the transformation tensor $A_{n}^{\mu}$ as follows:

$$
\left\langle e^{\mu}\right|=\left\langle e^{\mu} \mid a_{n}\right\rangle\left\langle a^{n}\right|=A_{n}^{\mu}\left\langle a^{n}\right| .
$$

The tensor $\psi^{\mu}$ in the basis $\left\{\left|e_{\mu}\right\rangle\right\}$ then transforms into the tensor $\psi^{n}$ in the basis $\left\{\left|a_{n}\right\rangle\right\}$ as

$$
\psi^{\mu}=A_{n}^{\mu} \psi^{n},
$$


and the tensor $H_{v}^{\mu}$ in the basis $\left\{\left|e_{\mu}\right\rangle\right\}$ then transforms into the tensor $H_{m}^{n}$ in the basis $\left\{\left|a_{n}\right\rangle\right\}$ as

$$
H_{v}^{\mu}=A_{n}^{\mu} H_{m}^{n} A_{v}^{m},
$$

where $A^{m}{ }_{v}$ defines the transformation

$$
\left|e_{\nu}\right\rangle=\left|a_{m}\right\rangle\left\langle a^{m} \mid e_{\nu}\right\rangle=\left|a_{m}\right\rangle A_{\nu}^{m} .
$$

The transformation tensors $A^{\mu}{ }_{n}$ and $A^{m}{ }_{v}$ are interrelated by

$$
\left\langle e^{\mu} \mid e_{\nu}\right\rangle=\left\langle e^{\mu} \mid a_{n}\right\rangle\left\langle a^{n} \mid e_{\nu}\right\rangle=A_{n}^{\mu} A^{n}{ }_{\nu}=\delta^{\mu}{ }_{\nu} ;
$$

i.e., they are inverse of each other.

In general, lower indices are referred to as covariant since they transform as the basis set, while upper indices are contravariant since they transform as the dual basis. Mathematical objects that do not transform following these rules are nontensors.

It is easy to show that the straight derivative of a first-rank tensor $\partial_{i} \psi^{\mu}$ is a nontensor:

$$
\partial_{i} \psi^{\mu}=\partial_{i}\left(A_{n}^{\mu} \psi^{n}\right)=\left(\partial_{i} A_{n}^{\mu}\right) \psi^{n}+A_{n}^{\mu} \partial_{i} \psi^{n},
$$

and therefore that $\partial_{i} \psi^{\mu} \neq A_{n}^{\mu} \partial_{i} \psi^{n}$, where there is clearly a term breaking the transformation rule.

The covariant derivative definition in Eq. (3) gives a well-behaved tensor since the defined $\partial_{i} \psi^{\mu}$ is the tensor representation of the vector $P_{\Omega} \partial_{i}\left(P_{\Omega}|\psi\rangle\right) \in \Omega$ :

$$
ð_{i} \psi^{\mu}=\left\langle e^{\mu}\right| \partial_{i}\left(P_{\Omega}|\psi\rangle\right)=A_{n}^{\mu}\left\langle a^{n}\right| \partial_{i}\left(P_{\Omega}|\psi\rangle\right)=A_{n}^{\mu}{\partial_{i}} \psi^{n} .
$$

If we want to check this using the definition in Eq. (4), which is a better definition in the sense that it only refers to quantities defined within $\Omega$, we need to start checking the transformation of Christoffel symbols under basis change, as follows:

$$
\begin{aligned}
D^{\mu}{ }_{\nu i} & =\left\langle e^{\mu}\left|\partial_{i}\right| e_{v}\right\rangle=A_{n}^{\mu}\left\langle a^{n}\right|\left(\partial_{i}\left|a_{m}\right\rangle A^{m}{ }_{v}\right) \\
& =A^{\mu}{ }_{n} D^{n}{ }_{m i} A^{m}{ }_{v}+A^{\mu}{ }_{n} \delta^{n}{ }_{m} \partial_{i} A^{m}{ }_{v},
\end{aligned}
$$

giving

$$
D_{\nu i}^{\mu}=A_{n}^{\mu} D_{m i}^{n} A_{v}^{m}+A_{m}^{\mu} \partial_{i} A^{m}{ }_{v},
$$

which shows that the Christoffel symbols are nontensors, as stated in the main text.

Now, for the transformation of $ð_{i} \psi^{\mu}$ under basis set change, we have

$$
\begin{aligned}
D_{\nu i}^{\mu} \psi^{\nu} & =A_{n}^{\mu} D_{m i}^{n} A_{\nu}^{m} \psi^{\nu}+A_{m}^{\mu}\left(\partial_{i} A^{m}{ }_{\nu}\right) \psi^{\nu} \\
& =A^{\mu}{ }_{n} D_{m i}^{n} \psi^{m}+A^{\mu}{ }_{m}\left(\partial_{i} A^{m}{ }_{\nu}\right) A^{\nu}{ }_{l} \psi^{l} .
\end{aligned}
$$

The last term contains the derivative of the inverse transformation, while Eq. (B1) contains the derivative of the direct transformation under basis change. In order to relate them, take the fact that $A^{\mu}{ }_{m} A^{m}{ }_{v}=\delta^{\mu}{ }_{\nu}$, and therefore

$$
\partial_{i}\left(A_{m}^{\mu} A_{v}^{m}\right)=\left(\partial_{i} A_{m}^{\mu}\right) A_{v}^{m}+A_{m}^{\mu}\left(\partial_{i} A_{v}^{m}\right)=0 .
$$

This gives us the sought after relation

$$
\partial_{i} A_{l}^{\mu}=-A_{m}^{\mu}\left(\partial_{i} A_{v}^{m}\right) A_{l}^{v} .
$$

Introducing Eq. (B5) into Eq. (B3), it follows that

$$
D_{\nu i}^{\mu} \psi^{\nu}=A_{n}^{\mu} D_{m i}^{n} \psi^{m}-\left(\partial_{i} A_{n}^{\mu}\right) \psi^{n} \text {. }
$$

Finally, the transformed covariant derivative would be the sum of Eqs. (B1) and (B6), giving

$$
ð_{i} \psi^{\mu}=A_{n}^{\mu} \partial_{i} \psi^{n}+A_{n}^{\mu} D_{m i}^{n} \psi^{m}=A_{n}^{\mu} \partial_{i} \psi^{n},
$$

which confirms the expected tensor character of the covariant derivative.

\section{APPENDIX C: STATE PROPAGATION}

Here we show the equivalence between Eqs. (7) and (8). For that, we may close Eq. (8) with $\left\langle e^{\mu}(\mathbf{R}+d \mathbf{R})\right|$ from the left, thereby obtaining

$$
\psi^{\mu}(\mathbf{R}+d \mathbf{R})=\left\langle e^{\mu}(\mathbf{R}+d \mathbf{R}) \mid e_{\nu}(\mathbf{R})\right\rangle\left\{\psi^{\nu}(\mathbf{R})+{\varlimsup_{i}}_{i} \psi^{\nu}(\mathbf{R}) d R^{i}\right\}
$$

(always keeping in mind that these relations are valid to linear order for infinitesimal $d \mathbf{R}$ ). Taking now the bra equivalent of Eq. (6), we have

$$
\left\langle e^{\mu}(\mathbf{R}+d \mathbf{R})\right|=\left\langle e^{\mu}(\mathbf{R})\right|+D^{\mu}{ }_{i \nu} d R^{i}\left\langle e^{\nu}(\mathbf{R})\right| .
$$

For $D_{i v}^{\mu}$ defined as in Eq. (D1), one then finds that

$$
\psi^{\mu}(\mathbf{R}+d \mathbf{R})=\left(\delta^{\mu}{ }_{\nu}+D^{\mu}{ }_{i \sigma} \delta^{\sigma}{ }_{\nu} d R^{i}\right)\left\{\psi^{\nu}+{ }_{i} \psi^{\nu} d R^{i}\right\},
$$

where the whole right-hand side refers to objects defined at $\mathbf{R}$. To linear order it gives

$$
\psi^{\mu}(\mathbf{R}+d \mathbf{R})=\psi^{\mu}+\left({\varlimsup_{i}} \psi^{\mu}+D_{i \nu}^{\mu} \psi^{\nu}\right) d R^{i} .
$$

Using now $D_{i v}^{\mu}=-D^{\mu}{ }_{v i}$ (see Appendix D), and the definition of the covariant derivative [Eq. (4)], the result is obtained

$$
\psi^{\mu}(\mathbf{R}+d \mathbf{R})=\psi^{\mu}(\mathbf{R})+\partial_{i} \psi^{\mu}(\mathbf{R}) d R^{i},
$$

which is Eq. (7), and shows how the vector $|\psi(\mathbf{R})\rangle=\psi^{\mu}(\mathbf{R})\left|e_{\mu}(\mathbf{R})\right\rangle \quad$ evolves into $\quad|\psi(\mathbf{R}+d \mathbf{R})\rangle=$ $\psi^{\mu}(\mathbf{R}+d \mathbf{R})\left|e_{\mu}(\mathbf{R}+d \mathbf{R})\right\rangle$.

\section{APPENDIX D: CHRISTOFFEL SYMBOL RELATIONS}

Christoffel symbols are related to their equivalent in representations beyond the natural one introduced in the main text [in Eqs. (5) and (11)]. The following can be defined:

$$
\begin{array}{ll}
D_{\nu i}^{\mu}=\left\langle e^{\mu} \mid \partial_{i} e_{\nu}\right\rangle, & D_{i v}^{\mu}=\left\langle\partial_{i} e^{\mu} \mid e_{\nu}\right\rangle, \\
D_{\mu i}{ }^{\nu}=\left\langle\partial_{i} e_{\mu} \mid e^{v}\right\rangle, & D_{\mu i}{ }^{\nu}=\left\langle e_{\mu} \mid \partial_{i} e^{v}\right\rangle, \\
D_{\mu v i}=\left\langle e_{\mu} \mid \partial_{i} e_{\nu}\right\rangle, & D_{\mu i v}=\left\langle\partial_{i} e_{\mu} \mid e_{\nu}\right\rangle, \\
D_{i}^{\mu \nu}=\left\langle\partial_{i} e^{\mu} \mid e^{v}\right\rangle, & D^{\mu \nu}{ }_{i}=\left\langle e^{\mu} \mid \partial_{i} e^{v}\right\rangle .
\end{array}
$$

A further set of eight would be defined for the contravariant derivatives, which would have an upper $i$ index, corresponding to $\partial^{i}=\partial / \partial R_{i}$. This is needed if the axes in the parameter space $\Theta$ are oblique. We will not consider that possibility here, although the extension would be straightforward using the metric in $\Theta$ space. For orthonormal axes, $\partial_{i}=\partial^{i}$.

These magnitudes relate to each other in different ways. The shifting identities shift the $i$ derivation from one Greek index to the other. They are obtained from expanding the derivative of the metric tensors, $\partial_{i} S_{\mu \nu}, \partial_{i} S^{\mu \nu}$, and from $\partial_{i} \delta^{\mu}{ }_{\nu}=\partial_{i} \delta_{\mu}{ }^{\nu}=0$, and result in the following:

$$
\begin{aligned}
& D_{\nu i}^{\mu}=-D_{i v}^{\mu}, \\
& D_{\mu i}^{{ }^{\nu}{ }_{i v}}=-D_{\mu i}{ }^{\nu},
\end{aligned}
$$




$$
\begin{aligned}
& D_{\mu v i}=-D_{\mu i v}+\partial_{i} S_{\mu \nu}, \\
& D^{\mu \nu}{ }_{i}=-D^{\mu \nu}{ }_{i}{ }^{\nu}+\partial_{i} S^{\mu \nu},
\end{aligned}
$$

the first one of them being repeated from Eq. (12).

A further set of relations is obtained simply by complex conjugation, i.e.,

$$
D_{\mu i \nu}=\left\langle\partial_{i} e_{\mu} \mid e_{\nu}\right\rangle=\left\langle e_{\nu} \mid \partial_{i} e_{\mu}\right\rangle^{*}=D_{\nu \mu i}^{*} .
$$

This gives rise to the following set:

$$
\begin{aligned}
& D_{\nu i}^{\mu}=D_{v i}^{\mu *}, \quad D_{i v}^{\mu}=D_{\nu i}^{\mu *}, \\
& D_{\mu \nu i}=D_{v i \mu}^{*}, \quad D_{i}^{\mu \nu}=D_{i}^{v \mu *} .
\end{aligned}
$$

Metric tensors can be used to raise or lower indices by contraction, but only for the indices in the symbols that do not refer to a derived basis or dual vector, i.e., for Greek indices that do not immediately precede a Latin index. For example,

$$
D_{\mu \nu i}=\left\langle e_{\mu} \mid \partial_{i} e_{\nu}\right\rangle=\left\langle e_{\mu} \mid e_{\sigma}\right\rangle\left\langle e^{\sigma} \mid \partial_{i} e_{\nu}\right\rangle=S_{\mu \sigma} D_{\nu i}^{\sigma} .
$$

The following relations follow these raising/lowering rules:

$$
\begin{aligned}
& D_{\nu i}^{\mu}=S^{\mu \sigma} D_{\sigma \nu i}, \quad D_{i \nu}^{\mu}=D_{i}^{\mu \sigma} S_{\sigma \nu}, \\
& D_{\mu i}{ }^{\nu}{ }_{i}=S_{\mu \sigma} D^{\sigma \nu}{ }_{i}, \quad D_{\mu i}{ }^{v}=D_{\mu i \sigma} S^{\sigma v} \text {, } \\
& D_{\mu \nu i}=S_{\mu \sigma} D_{\nu i}^{\sigma}, \quad D_{\mu i \nu}=D_{\mu i}{ }^{\sigma} S_{\sigma \nu}, \\
& D^{\mu \nu}{ }_{i}=S^{\mu \sigma} D_{\sigma i}{ }^{\nu}, \quad D^{\mu}{ }_{i}{ }^{\nu}=D_{i \sigma}^{\mu} S^{\sigma v} .
\end{aligned}
$$

If the Greek index to be lowered or raised is immediately followed by a Latin index, indicating a derivative, the relations get more complicated. See for instance

$$
\begin{aligned}
D_{\mu \nu i} & =\left\langle e_{\mu} \mid \partial_{i} e_{\nu}\right\rangle=\left\langle e_{\mu}\right| \partial_{i}\left\{\left|e^{\sigma}\right\rangle\left\langle e_{\sigma} \mid e_{\nu}\right\rangle\right\} \\
& =D_{\mu i}{ }^{\sigma} S_{\sigma \nu}+\delta_{\mu}{ }^{\sigma} \partial_{i} S_{\sigma \nu}=D_{\mu i}{ }^{\sigma} S_{\sigma \nu}+\partial_{i} S_{\mu \nu} .
\end{aligned}
$$

This illustrates the raising/lowering derived relations that follow

$$
\begin{aligned}
& D_{i}^{\mu \sigma} S_{\sigma \nu}=D^{\mu}{ }_{\nu i}-S^{\mu \sigma} \partial_{i} S_{\sigma \nu}, \\
& D_{\mu \sigma i} S^{\sigma v}=D_{\mu i}{ }^{v}-S_{\mu \sigma} \partial_{i} S^{\sigma v}, \\
& D_{\mu i}^{\sigma} S_{\sigma v}=D_{\mu \nu i}-\partial_{i} S_{\mu \nu}, \\
& D_{\sigma i}^{\mu} S^{\sigma v}=D^{\mu \nu}{ }_{i}-\partial_{i} S^{\mu \nu},
\end{aligned}
$$

for derived indices on the right, and

$$
\begin{aligned}
& S^{\mu \sigma} D_{\sigma i v}=D_{i v}^{\mu}-\left(\partial_{i} S^{\mu \sigma}\right) S_{\sigma \nu}, \\
& S_{\mu \sigma} D_{i}^{\sigma}{ }_{i}=D_{\mu i}{ }^{\nu}-\left(\partial_{i} S_{\mu \sigma}\right) S^{\sigma v}, \\
& S_{\mu \sigma} D_{i v}^{\sigma}=D_{\mu i v}-\partial_{i} S_{\mu \nu}, \\
& S^{\mu \sigma} D_{\sigma i}{ }^{\nu}=D_{i}^{\mu \nu}-\partial_{i} S^{\mu \nu},
\end{aligned}
$$

for derived indices on the left. If we now expand the derivative of the overlap, as in $\partial_{i} S_{\mu \nu}=D_{\mu i \nu}+D_{\mu \nu i}$, the following expressions are obtained from Eqs. (D5) and (D6):

$$
\begin{aligned}
& D_{i}^{\mu \sigma} S_{\sigma v}=-S^{\mu \sigma} D_{\sigma i v}, \\
& D_{\mu \sigma i} S^{\sigma v}=-S_{\mu \sigma} D^{\sigma}{ }_{i}{ }^{\nu}, \\
& D_{\mu i}^{\sigma} S_{\sigma v}=-D_{\mu i v}, \\
& D_{\sigma i}^{\mu} S^{\sigma v}=-D_{i}^{\mu \nu}, \\
& S_{\mu \sigma} D_{i v}^{\sigma}=-D_{\mu \nu i}, \\
& S^{\mu \sigma} D_{\sigma i}{ }^{\nu}=-D_{i}^{\mu \nu},
\end{aligned}
$$

the first two arising from the first two in both Eqs. (D5) and (D6), third and fourth from the last two in Eqs. (D5), and fifth and sixth from the last two in Eqs. (D6).

They are further related by metric tensors: Post-multiplying the third and fourth in Eqs. (D7) by $S^{\nu \lambda}$ and $S_{\nu \lambda}$, respectively [and using the regular raising/lowering of Eqs. (D4)] we recover the first two equations in Eqs. (D2), as we do by pre-multiplying the fifth and sixth by the same metric tensors. Pre- or post-multiplying by the appropriate metric tensor the first two equations in Eqs. (D7) give the last four equations of the same set.

The rules for derived raising and lowering are therefore as follows: (i) Only tensors with one upper and one lower Greek symbol can be directly contracted. (ii) They contract with a shift and a corresponding change of sign. (iii) The other tensors have to be first converted into the former type.

The last four equations in Eqs. (D7) reflect the first two rules (and are the only ones actually needed), while the first two reflect the third rule as follows. Say you need to contract $D^{\mu \sigma} S_{\sigma \nu}$. First you convert it to a contractible one, by lowering the first index (a derivativeless contraction), and then contract it with the second rule:

$$
D_{i}^{\mu \sigma} S_{\sigma v}=S^{\mu \lambda} D_{\lambda}^{\sigma}{ }_{i} S_{\sigma v}=-S^{\mu \lambda} D_{\lambda i v},
$$

which is the first equation of Eqs. (D7).

Finally, we derive within this context the relations $ð_{i} S_{\mu \nu}=$ $\mathrm{\partial}_{i} S^{\mu \nu}=0$. Doing it for the latter relation,

$$
ð_{i} S^{\mu \nu}=\partial_{i} S^{\mu \nu}+D_{\lambda i}^{\mu} S^{\lambda \nu}+S^{\mu \lambda} D_{\lambda i}{ }^{\nu} .
$$

This expression can be obtained from the covariant derivative of the tensor corresponding to the unity operator as in Eqs. (14) and (15), namely, from

$$
ð_{i} S^{\mu \nu}=\left\langle e^{\mu}\left|\partial_{i}\left\{P_{\Omega} 1 P_{\Omega}\right\}\right| e^{\nu}\right\rangle .
$$

Expanding now $\partial_{i} S^{\mu \nu}$ we obtain

$$
\partial_{i} S^{\mu \nu}=D_{i}^{\mu \nu}+D_{i}^{\mu \nu}
$$

Now for the last two terms of $ð S^{\mu \nu}$. Using the fourth and sixth equations in (D7) we get

$$
D_{\lambda i}^{\mu} S^{\lambda \nu}=-D_{i}^{\mu \nu} \text { and } S^{\mu \lambda} D_{\lambda i}{ }^{\nu}=-D_{i}^{\mu \nu} .
$$

Introducing the last three relations into the above expression for $ð_{i} S^{\mu \nu}$ we arrive at the expected result of $ð_{i} S^{\mu \nu}=0$. It can be similarly done for $ð_{i} S_{\mu \nu}$.

\section{APPENDIX E: CHAIN-RULE CONSISTENCY OF TENSORS}

Here we verify the following chain rule:

$$
ð_{i}\left(H_{\nu}^{\mu} \psi^{v}\right)=\left(ð_{i} H_{\nu}^{\mu}\right) \psi^{v}+H_{\nu}^{\mu}\left(ð_{i} \psi^{\nu}\right) .
$$

To see this, on the one hand we have

$$
\begin{aligned}
\partial_{i}\left(H_{\nu}^{\mu} \psi^{\nu}\right) & =\partial_{i}\left(H_{\nu}^{\mu} \psi^{\nu}\right)+D_{\lambda i}^{\mu}\left(H_{\nu}^{\lambda} \psi^{\nu}\right) \\
& =\left(\partial_{i} H^{\mu}{ }_{\nu}\right) \psi^{\nu}+H_{\nu}^{\mu}\left(\partial_{i} \psi^{\nu}\right)+D_{\lambda i}^{\mu} H_{\nu}^{\lambda} \psi^{\nu} .
\end{aligned}
$$

On the other hand,

$$
\left(ð_{i} H_{\nu}^{\mu}\right) \psi^{v}=\left(\partial_{i} H_{\nu}^{\mu}\right) \psi^{v}+D_{\lambda i}^{\mu} H_{\nu}^{\lambda} \psi^{v}-H_{\lambda}^{\mu} D_{\nu i}^{\lambda} \psi^{v}
$$

and

$$
H_{\nu}^{\mu}\left(ð_{i} \psi^{v}\right)=H_{v}^{\mu}\left(\partial_{i} \psi^{v}\right)+H_{\nu}^{\mu} D_{\lambda}^{\nu} \psi^{\lambda} .
$$


Summing the last two equations, we see that their respective last terms cancel, and the sum results equal to the previous one, proving our original assertion in Eq. (E1).

Similarly, for the scalar $E=\psi_{\mu} H_{\nu}^{\mu} \psi^{\nu}$ it can be shown that

$$
ð_{i} E=ð_{i}\left(\psi_{\mu} H_{\nu}^{\mu} \psi^{\nu}\right)=\partial_{i}\left(\psi_{\mu} H_{\nu}^{\mu} \psi^{\nu}\right)=\partial_{i} E,
$$

as expected of a zero-rank tensor. It can be easily seen that

$$
\begin{aligned}
& \left(\partial_{i} \psi_{\mu}\right) H_{\nu}^{\mu} \psi^{\nu}+\psi_{\mu}\left(\partial_{i} H_{\nu}^{\mu}\right) \psi^{v}+\psi_{\mu} H_{\nu}^{\mu}\left(\partial_{i} \psi^{\nu}\right) \\
& \quad=\left(\partial_{i} \psi_{\mu}\right) H_{\nu}^{\mu} \psi^{v}+\psi_{\mu}\left(\partial_{i} H_{\nu}^{\mu}\right) \psi^{\nu}+\psi_{\mu} H_{\nu}^{\mu}\left(\partial_{i} \psi^{\nu}\right),
\end{aligned}
$$

whereupon the four terms with Christoffel symbols implied in the left-hand side cancel each other.

\section{APPENDIX F: UNITARY PROPAGATION}

If an orthonormal set of state vectors defined in $\mathcal{H}$ would propagate in $\Theta$ preserving their mutual orthonormality, as under the action of a unitary propagator in $\mathcal{H}$, do state vectors defined in $\Omega(\mathbf{R})$ do the same? Let us consider time evolution in this case for simplicity in the notation, and let us start from the set $\left\{\left|\psi_{n}(t)\right\rangle \in \Omega(t)\right\}$ at a given time $t$, such that

$$
\left\langle\psi_{n}(t) \mid \psi_{m}(t)\right\rangle=\delta_{n m} .
$$

When evolving from $t$ to $t+d t$, using Eq. (7),

$$
\begin{aligned}
\left|\psi_{m}(t+d t)\right\rangle & =\left(\psi_{m}^{\mu}+\partial_{t} \psi_{m}^{\mu} d t\right)\left|e_{\mu}(t+d t)\right\rangle, \\
\left\langle\psi_{n}(t+d t)\right| & =\left(\psi_{n v}+\partial_{t} \psi_{n v} d t\right)\left\langle e^{v}(t+d t)\right| .
\end{aligned}
$$

Their scalar product gives (up to linear terms in $d t$ )

$$
\begin{aligned}
& \left\langle\psi_{n}(t+d t) \mid \psi_{m}(t+d t)\right\rangle \\
& \quad=\left(\psi_{n v}+\partial_{t} \psi_{n \nu} d t\right) \delta_{\mu}^{\nu}\left(\psi_{m}^{\mu}+\partial_{t} \psi_{m}^{\mu} d t\right) \\
& \quad=\psi_{\mu n} \psi_{m}^{\mu}+\left\{\left(\partial_{t} \psi_{\mu n}\right) \psi_{m}^{\mu}+\psi_{\mu n}\left(\partial_{t} \psi_{m}^{\mu}\right)\right\} d t \\
& \quad=\psi_{\mu n} \psi_{m}^{\mu}+\partial_{t}\left(\psi_{\mu n} \psi_{m}^{\mu}\right) d t .
\end{aligned}
$$

If we thus define unitary propagation as the one that keeps

$$
\partial_{t}\left(\psi_{\mu n} \psi_{m}^{\mu}\right)=\partial_{t}\left(\psi_{\mu n} \psi_{m}^{\mu}\right)=0,
$$

[it is easy to show that $\partial_{t}\left(\psi_{\mu n} \psi_{m}^{\mu}\right)=\partial_{t}\left(\psi_{\mu n} \psi_{m}^{\mu}\right)$, which makes sense being the scalar products zero-rank tensors] then

$$
\left\langle\psi_{n}(t+d t) \mid \psi_{m}(t+d t)\right\rangle=\left\langle\psi_{n}(t) \mid \psi_{m}(t)\right\rangle,
$$

and, therefore, orthonormal vectors stay orthonormal.

\section{APPENDIX G: LINEARIZATION OF BASIS TRANSFORMATION}

Here we show that the expressions in Eqs. (19) and (7) for the propagation of a state vector are linearly equivalent. One can see the latter as a linearization of the former as follows. Taking the linear evolution of the bra $\left\langle e^{\mu}(\mathbf{R}+d \mathbf{R})\right|$, as in Eq. (C1), one finds that

$$
\begin{aligned}
A^{\mu}{ }_{\nu}(\mathbf{R}+d \mathbf{R}: \mathbf{R}) & =\left(\delta^{\mu}{ }_{\sigma}+D^{\mu}{ }_{i \sigma} d R^{i}\right)\left\langle e^{\sigma}(\mathbf{R}) \mid e_{\nu}(\mathbf{R})\right\rangle \\
& =\delta^{\mu}{ }_{\nu}+D^{\mu}{ }_{i \nu} d R^{i},
\end{aligned}
$$

and, plugging it into Eq. (19), that

$$
\begin{aligned}
\psi^{\mu}(\mathbf{R}+d \mathbf{R}) & =\left(\delta^{\mu}{ }_{\nu}+D^{\mu}{ }_{i \nu} d R^{i}\right)\left\{\psi^{\nu}+\left(ð_{i} \psi^{\nu}\right) d R^{i}\right\} \\
& =\psi^{\mu}+\left({\varlimsup_{i}} \psi^{\mu}+D^{\mu}{ }_{i \nu} \psi^{\nu}\right) d R^{i}
\end{aligned}
$$

(dropping terms in $d R^{i}$ beyond linear in the latter equation). Using that $D_{i v}^{\mu}=-D_{\nu i}^{\mu}$ [Eq. (12) and see also Appendix $\mathrm{D}]$, and remembering the definition of the covariant derivative [Eq. (4)], we recover Eq. (7).

\section{APPENDIX H: BASIS ROTATION AND ANTI-HERMITIAN $D_{\mu \nu i}$}

Here we show how a transformation of the basis corresponding to a small rotation in $\Omega$ is described by an anti-Hermitian $D_{\mu v i}$ tensor. By a small rotation we mean a basis transformation from the set $\left\{\left|e_{\mu}(\mathbf{R})\right\rangle\right\}$ to the set $\left\{\left|e_{\mu}(\mathbf{R}+d \mathbf{R})\right\rangle\right\}$, such that

$$
S_{\mu \nu}(\mathbf{R}+d \mathbf{R})=S_{\mu \nu}(\mathbf{R})
$$

i.e., all the scalar products are maintained. Expanding the lefthand side, we find that

$$
\begin{aligned}
& S_{\mu \nu}(\mathbf{R}+d \mathbf{R})=\left\langle e_{\mu}(\mathbf{R}+d \mathbf{R}) \mid e_{\nu}(\mathbf{R}+d \mathbf{R})\right\rangle \\
& \quad=\left\langle e_{\mu}(\mathbf{R}+d \mathbf{R}) \mid e^{\sigma}(\mathbf{R})\right\rangle S_{\sigma \lambda}(\mathbf{R})\left\langle e^{\lambda}(\mathbf{R}) \mid e_{\nu}(\mathbf{R}+d \mathbf{R})\right\rangle .
\end{aligned}
$$

Now, to first order in the transformation, we have

$$
\begin{aligned}
\left|e_{\nu}(\mathbf{R}+d \mathbf{R})\right\rangle & \simeq\left|e_{\nu}\right\rangle+\left|e_{\kappa}\right\rangle\left\langle e^{\kappa} \mid \partial_{i} e_{\nu}\right\rangle d R^{i} \\
& =\left|e_{\nu}\right\rangle+\left|e_{\kappa}\right\rangle D^{\kappa}{ }_{\nu i} d R^{i},
\end{aligned}
$$

where all the right-hand-side terms are evaluated at $\mathbf{R}$ Therefore,

$$
\left\langle e^{\lambda}(\mathbf{R}) \mid e_{v}(\mathbf{R}+d \mathbf{R})\right\rangle \simeq \delta_{v}^{\lambda}+D^{\lambda}{ }_{\nu i} d R^{i},
$$

and, similarly,

$$
\left\langle e_{\mu}(\mathbf{R}+d \mathbf{R}) \mid e^{\sigma}(\mathbf{R})\right\rangle \simeq \delta_{\mu}{ }^{\sigma}+D_{\mu i}{ }^{\sigma} d R^{i} .
$$

Introducing the last two expressions in Eq. (H1), we obtain

$$
S_{\mu \nu}(\mathbf{R}+d \mathbf{R}) \simeq\left(\delta_{\mu}{ }^{\sigma}+D_{\mu i}{ }^{\sigma} d R^{i}\right) S_{\sigma \lambda}\left(\delta_{\nu}^{\lambda}+D_{\nu i}^{\lambda} d R^{i}\right),
$$

which, to first order, reads

$$
S_{\mu \nu}(\mathbf{R}+d \mathbf{R}) \simeq S_{\mu \nu}+\left\{D_{\mu i}{ }^{\sigma} S_{\sigma \nu}+S_{\mu \lambda} D_{\nu i}^{\lambda}\right\} d R^{i} .
$$

Therefore, the overlap conservation premise for pure basis rotations is kept if

$$
D_{\mu i}{ }^{\sigma} S_{\sigma \nu}=-S_{\mu \lambda} D_{\nu i}^{\lambda}
$$

or, using the overlaps to lower the indices,

$$
D_{\mu i v}=-D_{\mu \nu i},
$$

which is equivalent to

$$
D_{\mu \nu i}=-D_{\nu \mu i}^{*} .
$$

The last three expressions are different forms that reflect the fact that the $D$ tensor should be anti-Hermitian for the overlap to be conserved, as had been proposed.

\section{APPENDIX I: INVERSE SECOND-RANK TENSORS}

Here, we establish the notation for the inverse of secondrank tensors, as used in the discussion of the different integrators. They conform with inverse (square) matrices for the matrix representation of such tensors, but they generalize to 
other representations. For the sake of completeness we define the inverse from scratch.

Drawing from square matrices, if we have a tensor $A_{\mu \nu}$ acting on vectors in $\Omega$, we will define its inverse tensor as the tensor $B^{\mu \nu}$ that fulfils

$$
A_{v \sigma} B^{\sigma \mu}=B^{\mu \sigma} A_{\sigma v}=\delta_{v}^{\mu}
$$

where we have chosen to indicate the indices of the inverse tensor in this representation as both up. In addition to being parallel to the metric tensor corresponding to $S^{-1}$, this choice makes sense if we see this equation as

$$
\left\langle e_{\nu}|A| e_{\sigma}\right\rangle\left\langle e^{\sigma}|B| e^{\mu}\right\rangle=\left\langle e^{\mu}|B| e^{\sigma}\right\rangle\left\langle e_{\sigma}|A| e_{\nu}\right\rangle=\delta_{v}^{\mu},
$$

which is nothing but

$$
P_{\Omega} A P_{\Omega} B P_{\Omega}=P_{\Omega} B P_{\Omega} A P_{\Omega}=P_{\Omega},
$$

or

$$
\left(P_{\Omega} A P_{\Omega}\right)\left(P_{\Omega} B P_{\Omega}\right)=\left(P_{\Omega} B P_{\Omega}\right)\left(P_{\Omega} A P_{\Omega}\right)=P_{\Omega},
$$

which is the definition of the inverse for operators defined as acting within $\Omega$.

Equation (I1) allows us to use conventional matrix inversion procedures to obtain $\underline{B}$ from $\underline{A}$. Equation (I2) allows us to extend the definition of the inverse tensor to other representations, remembering that $P_{\Omega}$ is both $\left|e_{\mu}\right\rangle\left\langle e^{v}\right|$ and $\left|e^{\mu}\right\rangle\left\langle e_{\nu}\right|$, thus obtaining

$$
\begin{aligned}
A_{\sigma}^{\mu} B^{\sigma}{ }_{\nu} & =B^{\mu}{ }_{\sigma} A_{\nu}^{\sigma}=\delta_{\nu}^{\mu}, \\
A_{\mu}{ }^{\sigma} B_{\sigma}{ }^{v} & =B_{\mu}{ }^{\sigma} A_{\sigma}{ }^{\nu}=\delta_{\mu}^{v}, \\
A^{\mu \sigma} B_{\sigma \nu} & =B_{\nu \sigma} A^{\sigma \mu}=\delta_{\nu}^{\mu}, \\
A_{\mu \sigma} B^{\sigma v} & =B^{\nu \sigma} A_{\sigma \mu}=\delta_{\mu}^{v}
\end{aligned}
$$

[the latter being Eq. (I1)], plus other possibilities like

$$
\begin{aligned}
A_{\mu}{ }^{\sigma} S_{\sigma \kappa} B^{\kappa}{ }_{\nu} & =S_{\mu \nu}, \\
B_{\mu \sigma} A^{\sigma}{ }_{\nu} & =S_{\mu \nu} .
\end{aligned}
$$

Many other expressions can be obtained from Eq. (I2), or derived from each other by using metric tensors to raise or lower indices, which applies to both $A$ and $B$ tensors, as representations of well-defined operators in the abstract Hilbert space.

As for notation, we will denote the elements of the inverse of $A_{\mu \nu}$ taken as a matrix as

$$
A^{-1 \mu \nu}=\left(A^{-1}\right)^{\mu \nu}=\left(A_{\mu \nu}\right)^{-1},
$$

corresponding to the matrix representation. Note the different position of the indices depending on whether inside or outside the inversion, a feature which has significant relevance in the calculation of energy gradients using nonorthogonal functions [37]. For the natural representation, the inverse of $A^{\mu}{ }_{v}$ will be represented by

$$
A^{-1 \mu}{ }_{\nu}=\left(A^{-1}\right)_{\nu}^{\mu}=\left(A_{\mu}^{\nu}\right)^{-1} .
$$

\section{APPENDIX J: MODIFIED CRANK-NICHOLSON INTEGRATOR}

Here we derive Eq. (36),

$$
\begin{aligned}
\psi^{\mu}(t+d t)= & {\left[S_{\mu \lambda}(t+d t)+i \frac{d t}{2} H_{\mu \lambda}(t+d t)\right]^{-1} } \\
& \times A_{\lambda}^{\sigma}(t+d t: t)\left\{S_{\sigma \nu}(t)-i \frac{d t}{2} H_{\sigma v}(t)\right\} \psi^{v}(t) .
\end{aligned}
$$

Beginning with Eq. (19), we may first transform to the matrix representation

$$
\begin{aligned}
\psi^{\mu}(t+d t) & =A^{\mu}{ }_{\nu}(t+d t: t)\left\{\psi^{v}+\left(ð_{t} \psi^{v}\right) d t\right\} \\
& =A^{\mu \sigma}(t+d t: t) S_{\sigma v}\left\{\psi^{v}+\left(ð_{t} \psi^{v}\right) d t\right\}
\end{aligned}
$$

and then, using Eq. (30), replace the $S_{\sigma \nu} \partial_{t} \psi^{\nu}$ term by $-i H_{\sigma \nu} \psi^{\nu}$ to solve the Schrödinger equation:

$$
\begin{aligned}
\psi^{\mu}(t+d t)= & A^{\mu \sigma}(t+d t: t) \\
& \times\left\{S_{\sigma \nu}(t) \psi^{\nu}(t)-i H_{\sigma \nu}(t) \psi^{\nu}(t) d t\right\} .
\end{aligned}
$$

Invoking the Crank-Nicholson idea of forward and backward time propagation, as in Eq. (31), we may write to first order that

$$
\begin{aligned}
\psi^{\kappa}\left(t+\frac{d t}{2}\right)= & A^{\kappa \sigma}\left(t+\frac{d t}{2}: t\right) \\
& \times\left\{S_{\sigma \nu}(t)-i \frac{d t}{2} H_{\sigma \nu}(t)\right\} \psi^{\nu}(t), \\
\psi^{\kappa}\left(t+\frac{d t}{2}\right)= & A^{\kappa \lambda}\left(t+\frac{d t}{2}: t+d t\right) \\
& \times\left\{S_{\lambda \mu}(t+d t)+i \frac{d t}{2} H_{\lambda \mu}(t+d t)\right\} \\
& \times \psi^{\mu}(t+d t) .
\end{aligned}
$$

We then get rid of $\psi^{\kappa}(t+d t / 2)$ and use the following relations: (i) inversion

$$
A^{\mu \sigma}\left(t+\frac{d t}{2}: t\right) A_{\sigma \nu}\left(t: t+\frac{d t}{2}\right)=\delta^{\mu}{ }_{\nu}
$$

and (ii) two-step propagation

$$
A_{\lambda \kappa}\left(t+d t: t+\frac{d t}{2}\right) A^{\kappa \sigma}\left(t+\frac{d t}{2}: t\right)=A_{\lambda}{ }^{\sigma}(t+d t: t),
$$

which lead to Eq. (36).

\section{APPENDIX K: ADEQUACY OF LÖWDIN-BASED PROPAGATION}

\section{General considerations}

The key question is to what extent the propagation given by the Löwdin orthogonalization procedure, Eq. (40), faithfully integrates the time-dependent Schrödinger equation of motion in Eq. (29). Section III A 4 shows a clear counterexample, demonstrating that it is not the case in general. Here we get a bit deeper into the question. 
The correct time propagation is defined by

$$
\begin{aligned}
\psi^{\mu}(t+d t) & =\psi^{\mu}(t)+\partial_{t} \psi^{\mu}(t) d t \\
& =\psi^{\mu}(t)+\left\{\partial_{t} \psi^{\mu}(t)-D^{\mu}{ }_{\nu t}(t) \psi^{\nu}(t)\right\} d t,
\end{aligned}
$$

the latter being a linearization of

$$
\psi^{\mu}(t+d t)=A_{v}^{\mu}(t+d t: t)\left\{\psi^{v}+\partial_{t} \psi^{v} d t\right\},
$$

which accounts for the basis set transformation between $t$ and $t+d t$, as in Eq. (19). The question is now whether the above coincides with

$$
\psi^{\mu}(t+d t)=S^{-1 / 2 \mu \lambda}(t+d t) S_{\lambda \nu}^{1 / 2}(t)\left\{\psi^{\nu}+ð_{t} \psi^{\nu} d t\right\}
$$

to linear order in $d t$. To that order we can expand $S^{-1 / 2 \mu \lambda}(t+$ $d t$ ) as follows:

$$
S^{-1 / 2 \mu \lambda}(t+d t)=S^{-1 / 2 \mu \lambda}(t)+\partial_{t} S^{-1 / 2 \mu \lambda}(t) d t,
$$

thereby obtaining, also to linear order,

$$
\begin{aligned}
& S^{-1 / 2 \mu \lambda}(t+d t) S_{\lambda \nu}^{1 / 2}(t) \\
& \quad=\delta_{\nu}^{\mu}+\partial_{t} S^{-1 / 2 \mu \lambda}(t) S_{\lambda \nu}^{1 / 2}(t) d t,
\end{aligned}
$$

and Eq. (K2) becomes, again to linear order,

$$
\psi^{\mu}(t+d t)=\psi^{\mu}(t)+\left\{ð_{t} \psi^{\mu}(t)-G^{\mu}{ }_{\nu t}(t) \psi^{\nu}(t)\right\} d t,
$$

where we have defined

$$
G^{\mu}{ }_{v t} \equiv-\partial_{t} S^{-1 / 2 \mu \lambda} S_{\lambda v}^{1 / 2} .
$$

Comparing then Eqs. (K1) and (K3), it becomes clear that the desired evolution of $\psi^{\mu}$ is recovered if

$$
G^{\mu}{ }_{\nu t}=D^{\mu}{ }_{v t},
$$

which is generally not the case, as inferred by the orthonormal evolution explained above, and is clear in some (not all) of the examples we present below.

\section{Examples}

Consider in the following a two-dimensional $\Omega$ Hilbert space, spanned by two basis vectors that we take as reference for $t=0$ :

$$
\left|e_{1}(0)\right\rangle=\left|e_{1}\right\rangle \text { and }\left|e_{2}(0)\right\rangle=\left|e_{2}\right\rangle .
$$

Starting with the illustration of the quite generic argument at the end of Sec. III A 4, we consider the evolving basis set

$$
\begin{aligned}
& \left|e_{1}(t)\right\rangle=\cos \theta(t)\left|e_{1}\right\rangle+\sin \theta(t)\left|e_{2}\right\rangle, \\
& \left|e_{2}(t)\right\rangle=-\sin \theta(t)\left|e_{1}\right\rangle+\cos \theta(t)\left|e_{2}\right\rangle,
\end{aligned}
$$

which is orthonormal at all times if it is for $t=0$. It is easy to check that

$$
D_{\bullet t}^{\bullet}=\left(\begin{array}{cc}
0 & -\partial_{t} \theta \\
\partial_{t} \theta & 0
\end{array}\right) \neq G_{\bullet t}^{\bullet}=\left(\begin{array}{ll}
0 & 0 \\
0 & 0
\end{array}\right),
$$

the latter resulting from $S_{\bullet \bullet}=S^{-1} \bullet=S^{1 / 2} \bullet=S^{-1 / 2} \bullet \bullet=\mathbb{1}$ and $\partial_{t} S^{-1 / 2} \bullet \bullet=\mathbb{D}$ at all times.

A similarly simple example is one in which the basis vectors are orthogonal, and only change norm with time, a particular case of what this formalism should cope with. Take then

$$
\begin{aligned}
& \left|e_{1}(t)\right\rangle=\alpha_{1}(t)\left|e_{1}\right\rangle, \\
& \left|e_{2}(t)\right\rangle=\alpha_{2}(t)\left|e_{2}\right\rangle .
\end{aligned}
$$

Then,

$$
\begin{array}{ll}
S_{\bullet \bullet}(t)=\left(\begin{array}{cc}
\alpha_{1}^{2} & 0 \\
0 & \alpha_{2}^{2}
\end{array}\right), & S^{-1 \bullet \bullet}(t)=\left(\begin{array}{cc}
\alpha_{1}^{-2} & 0 \\
0 & \alpha_{2}^{-2}
\end{array}\right), \\
S_{\bullet \bullet}^{1 / 2}(t)=\left(\begin{array}{cc}
\alpha_{1} & 0 \\
0 & \alpha_{2}
\end{array}\right), & S^{-1 / 2 \bullet \bullet}(t)=\left(\begin{array}{cc}
\alpha_{1}^{-1} & 0 \\
0 & \alpha_{2}^{-1}
\end{array}\right),
\end{array}
$$

and

$$
\begin{aligned}
\left\langle e^{1}(t)\right| & =\alpha_{1}^{-1}(t)\left\langle e^{1}\right|, \\
\left\langle e^{2}(t)\right| & =\alpha_{2}^{-1}(t)\left\langle e^{2}\right| .
\end{aligned}
$$

The tensor $D_{\nu t}^{\mu}$ then becomes

$$
D_{\bullet t}^{\bullet}=\left(\begin{array}{cc}
\frac{\partial_{t} \alpha_{1}}{\alpha_{1}} & 0 \\
0 & \frac{\partial_{t} \alpha_{2}}{\alpha_{2}}
\end{array}\right),
$$

and $G_{v t}^{\mu}=-\partial_{t} S^{-1 / 2 \mu l} S_{l v}^{1 / 2}$ becomes

$$
G_{\bullet}^{\bullet}=-\left(\begin{array}{cc}
\frac{-\partial_{t} \alpha_{1}}{\alpha_{1}^{2}} & 0 \\
0 & \frac{-\partial_{t} \alpha_{2}}{\alpha_{2}^{2}}
\end{array}\right)\left(\begin{array}{cc}
\alpha_{1} & 0 \\
0 & \alpha_{2}
\end{array}\right)=D^{\bullet} \bullet .
$$

In this case, the condition is fulfilled, and the propagator would thus be faithful.

Finally, let us consider both basis vectors normalized but with a mutual (real) overlap, $s(t)$, so that

$$
S_{\bullet \bullet}=\left(\begin{array}{ll}
1 & s \\
s & 1
\end{array}\right) \text { and } S^{-1} \bullet=\frac{1}{1-s^{2}}\left(\begin{array}{rr}
1 & -s \\
-s & 1
\end{array}\right) \text {. }
$$

By diagonalizing the metric as

$$
S_{\bullet \bullet}=\frac{1}{2}\left(\begin{array}{rr}
1 & 1 \\
1 & -1
\end{array}\right)\left(\begin{array}{cc}
1+s & 0 \\
0 & 1-s
\end{array}\right)\left(\begin{array}{rr}
1 & 1 \\
1 & -1
\end{array}\right),
$$

one readily obtains

$$
\begin{aligned}
S_{\bullet \bullet}^{1 / 2} & =\frac{1}{2}\left(\begin{array}{cc}
a+b & a-b \\
a-b & a+b
\end{array}\right), \\
S^{-1 / 2} \bullet & =\frac{1}{2}\left(\begin{array}{cc}
a^{-1}+b^{-1} & a^{-1}-b^{-1} \\
a^{-1}-b^{-1} & a^{-1}+b^{-1}
\end{array}\right), \\
\partial_{t} S^{-1 / 2} \bullet \bullet & =-\frac{\partial_{t} s}{4}\left(\begin{array}{cc}
a^{-3}-b^{-3} & a^{-3}+b^{-3} \\
a^{-3}+b^{-3} & a^{-3}-b^{-3}
\end{array}\right),
\end{aligned}
$$

where $a \equiv \sqrt{1+s}$ and $b \equiv \sqrt{1-s}$. Using the definition of $G^{\mu}{ }_{v t}$ of Eq. (K4) and operating, one gets

$$
G_{\bullet t}^{\bullet}=-\frac{\partial_{t} s}{2} \frac{1}{1-s^{2}}\left(\begin{array}{rr}
s & -1 \\
-1 & s
\end{array}\right),
$$

which incidentally is equal to $S^{-1} \mu \sigma \partial_{t} S_{\sigma \nu} / 2$, the expression we obtain by differentiating Eq. (K4) as if $S$ were a scalar.

We turn now to $D_{\nu t}^{\mu}$, and we build it from $D_{\mu v t}=$ $\left\langle e_{\mu}\left|\partial_{t}\right| e_{\nu}\right\rangle$. Let us assume that our two basis states correspond to, say, two $s$-like atomic orbitals as we would have for a 
minimal-basis description of $\mathrm{H}_{2}$. In that case $D_{11 t}=D_{22 t}=$ 0 , since the derivative of an $s$ orbital is a function of $p$ character, orthogonal to the original $s$. Remembering now that $s=\left\langle e_{1} \mid e_{2}\right\rangle$,

$$
\partial_{t} s=\left\langle e_{1} \mid \partial_{t} e_{2}\right\rangle+\left\langle\partial_{t} e_{1} \mid e_{2}\right\rangle=D_{12 t}+D_{21 t} .
$$

Let us now make a final assumption, which will turn out to be important: let us assume that the motion respects the center of symmetry, such that both states move equally towards or away from each other. In this case $D_{12 t}=D_{21 t}$. Putting it all together we have

$$
D_{\bullet \bullet t}=\left(\begin{array}{cc}
0 & \partial_{t} s / 2 \\
\partial_{t} s / 2 & 0
\end{array}\right),
$$

and since $D_{v t}^{\mu}=S^{\mu \sigma} D_{\sigma v t}$,

$$
D_{\bullet t}^{\bullet}=\frac{\partial_{t} s}{2} \frac{1}{1-s^{2}}\left(\begin{array}{rr}
1 & -s \\
-s & 1
\end{array}\right)\left(\begin{array}{ll}
0 & 1 \\
1 & 0
\end{array}\right),
$$

which is exactly the same as found for $G^{\bullet}{ }_{\bullet t}$ in Eq. (K5) above, finding thus another example for the Löwdin orthogonalization procedure working fine.

Interestingly, however, if instead of considering the symmetric motion we have orbital 1 moving towards a fixed orbital 2 , while keeping the same $s(t)$, then

$$
D_{\bullet \bullet t}=\left(\begin{array}{cc}
0 & 0 \\
\partial_{t} s & 0
\end{array}\right)
$$

and

$$
D_{\bullet t}^{\bullet}=\frac{\partial_{t} s}{1-s^{2}}\left(\begin{array}{rr}
-s & 0 \\
1 & 0
\end{array}\right) \neq G_{\bullet t}^{\bullet},
$$

since $G^{\mu}{ }_{v t}$ does not change from Eq. (K5).

It is interesting to note that the difference between Eqs. (K6) and (K7) is an anti-Hermitian $D_{\bullet \bullet t}$ tensor corresponding to a pure rotation of the basis in $\Omega$ (see Appendix $H$ ), which corresponds to a change of reference frame (Galilean transform) in real space for the moving atoms.

For an $N$-dimensional $\Omega$ space there are $N^{2}$ degrees of freedom in the set of anti-Hermitian matrices [corresponding to the dimension of the $U(N)$ group of unitary matrices]. There would then be $N^{2}$ possible ways to rotate in $\Omega$ without altering the metric tensors. Interestingly, however, for a basis of atomic-like orbitals moving with atoms, there are only four such dimensions, that correspond to the three translations in real space plus a global phase. All other possible rotations in $\Omega$ are not compatible with maintaining the shape of the atomic-like orbitals. [Only in the case of a basis made purely of $s$ orbitals, the three possible rotations in real (3D) space would also keep the metric tensors invariant.] Such a translation is the difference between Eqs. (K6) and (K7). Two key questions remain: (i) How relevant is the difference for the physical evolution? (ii) Can such a translation make the $G^{\mu}{ }_{v t}$ and $D^{\mu}{ }_{v t}$ tensors agree in general (as they do in the example above)? The answer to the latter seems likely to be negative since $4<N^{2}$ for systems with more than two basis states. These points should be studied in future work.
[1] M. J. Frisch, G. W. Trucks, H. B. Schlegel, G. E. Scuseria, M. A. Robb, J. R. Cheeseman, G. Scalmani, V. Barone, B. Mennucci, G. A. Petersson, H. Nakatsuji, M. Caricato, X. Li, H. P. Hratchian, A. F. Izmaylov, J. Bloino, G. Zheng, J. L. Sonnenberg, M. Hada, M. Ehara, K. Toyota, R. Fukuda, J. Hasegawa, M. Ishida, T. Nakajima, Y. Honda, O. Kitao, H. Nakai, T. Vreven, J. A. Montgomery, Jr., J. E. Peralta, F. Ogliaro, M. Bearpark, J. J. Heyd, E. Brothers, K. N. Kudin, V. N. Staroverov, R. Kobayashi, J. Normand, K. Raghavachari, A. Rendell, J. C. Burant, S. S. Iyengar, J. Tomasi, M. Cossi, N. Rega, J. M. Millam, M. Klene, J. E. Knox, J. B. Cross, V. Bakken, C. Adamo, J. Jaramillo, R. Gomperts, R. E. Stratmann, O. Yazyev, A. J. Austin, R. Cammi, C. Pomelli, J. W. Ochterski, R. L. Martin, K. Morokuma, V. G. Zakrzewski, G. A. Voth, P. Salvador, J. J. Dannenberg, S. Dapprich, A. D. Daniels, O. Farkas, J. B. Foresman, J. V. Ortiz, J. Cioslowski, and D. J. Fox, Gaussian-09 Revision E.01 (Gaussian Inc., Wallingford, CT, 2009).

[2] R. Ahlrichs, M. Bär, M. Häser, H. Horn, and C. Kölmel, Chem. Phys. Lett. 162, 165 (1989).

[3] M. S. Gordon and M. W. Schmidt, in Theory and Applications of Computational Chemistry: The First Forty Years, edited by C. E. Dykstra, G. Frenking, K. S. Kim, and G. E. Scuseria (Elsevier, Amsterdam, 2005), pp. 1167-1189.

[4] S. van Gisbergen, J. Snijders, and E. Baerends, Comput. Phys. Commun. 118, 119 (1999).
[5] J. M. Soler, E. Artacho, J. D. Gale, A. Garcia, J. Junquera, P. Ordejon, and D. Sanchez-Portal, J. Phys.: Condens. Matter 14, 2745 (2002).

[6] J. VandeVondele, M. Krack, F. Mohamed, M. Parrinello, T. Chassaing, and J. Hutter, Comput. Phys. Commun. 167, 103 (2005).

[7] R. Dovesi, R. Orlando, A. Erba, C. M. Zicovich-Wilson, B. Civalleri, S. Casassa, L. Maschio, M. Ferrabone, M. De La Pierre, P. D’Arco, Y. Noël, M. Causà, M. Rérat, and B. Kirtman, Int. J. Quantum Chem. 114, 1287 (2014).

[8] T. Ozaki, Phys. Rev. B 67, 155108 (2003).

[9] V. Blum, R. Gehrke, F. Hanke, P. Havu, V. Havu, X. Ren, K. Reuter, and M. Scheffler, Comput. Phys. Commun. 180, 2175 (2009).

[10] C.-K. Skylaris, P. D. Haynes, A. A. Mostofi, and M. C. Payne, J. Chem. Phys. 122, 084119 (2005).

[11] D. R. Bowler and T. Miyazaki, Rep. Prog. Phys. 75, 036503 (2012).

[12] S. Mohr, L. E. Ratcliff, L. Genovese, D. Caliste, P. Boulanger, S. Goedecker, and T. Deutsch, Phys. Chem. Chem. Phys. 17, 31360 (2015).

[13] P. O. Löwdin, J. Chem. Phys. 18, 365 (1950).

[14] E. Runge and E. K. U. Gross, Phys. Rev. Lett. 52, 997 (1984).

[15] K. Yabana and G. F. Bertsch, Phys. Rev. B 54, 4484 (1996).

[16] A. Tsolakidis, D. Sanchez-Portal, and R. M. Martin, Phys. Rev. B 66, 235416 (2002). 
[17] X. Andrade, D. Strubbe, U. D. Giovannini, A. H. Larsen, M. J. T. Oliveira, J. Alberdi-Rodriguez, A. Varas, I. Theophilou, N. Helbig, M. J. Verstraete, L. Stella, F. Nogueira, A. Aspuru-Guzik, A. Castro, M. A. L. Marques, and A. Rubio, Phys. Chem. Chem. Phys. 17, 31371 (2015).

[18] C. Y. Yam, S. Yokojima, and G. H. Chen, Phys. Rev. B 68, 153105 (2003).

[19] C. O'Rourke and D. R. Bowler, J. Chem. Phys. 143, 102801 (2015).

[20] T. N. Todorov, J. Phys.: Condens. Matter 13, 10125 (2001).

[21] T. Kunert and R. Schmidt, Eur. Phys. J. D 25, 15 (2003).

[22] G. Kolesov, O. Granäs, R. Hoyt, D. Vinichenko, and E. Kaxiras, J. Chem. Theory Comput. 12, 466 (2016).

[23] S. Meng and E. Kaxiras, J. Chem. Phys. 129, 054110 (2008).

[24] G. Cui, W. Fang, and W. Yang, Phys. Chem. Chem. Phys. 12, 416 (2010).

[25] P. Pulay, Mol. Phys. 17, 197 (1969).

[26] D. Vanderbilt and J. D. Joannopoulos, Phys. Rev. B 22, 2927 (1980).

[27] L. E. Ballantine and M. Kolá, J. Phys. C 19, 981 (1986).

[28] E. Artacho and L. Miláns del Bosch, Phys. Rev. A 43, 5770 (1991).

[29] M. Head-Gordon, P. E. Maslen, and C. A. White, J. Chem. Phys. 108, 616 (1998).

[30] H. G. Hiscock and A. J. W. Thom, J. Chem. Theory Comput. 10, 4795 (2014).

[31] D. D. O'Regan, M. C. Payne, and A. A. Mostofi, Phys. Rev. B 83, 245124 (2011).

[32] M. Soriano and J. J. Palacios, Phys. Rev. B 90, 075128 (2014).

[33] D. Jacob, J. Phys.: Condens. Matter 27, 245606 (2015).

[34] J. D. Weeks, P. W. Anderson, and A. G. H. Davidson, J. Chem. Phys. 58, 1388 (1973).

[35] D. W. Bullett, J. Phys. C 8, 2695 (1975).

[36] P. W. Anderson, Phys. Rep. 110, 311 (1984).
[37] C. A. White, P. Maslen, M. S. Lee, and M. Head-Gordon, Chem. Phys. Lett. 276, 133 (1997).

[38] M. Head-Gordon, Y. Shao, C. Saravanan, and C. A. White, Mol. Phys. 101, 37 (2003).

[39] D. D. O'Regan, Optimised Projections for the Ab Initio Simulation of Large and Strongly Correlated Systems, Springer Theses XVI (Springer Verlag, Berlin, 2012).

[40] L. P. Lebedev, M. J. Cloud, and V. A. Eremeyev, Tensor Analysis with Applications to Mechanics (World Scientific, Singapore, 2010), p. 12.

[41] A. Einstein, Ann. Phys. 354, 769 (1916).

[42] W. Kohn and L. J. Sham, Phys. Rev. 140, A1133 (1965).

[43] D. Husemöller, Fibre Bundles, 3rd ed., Graduate Texts in Mathematics, Vol. 20 (Springer Verlag, New York, 1994).

[44] M. V. Berry, Proc. R. Soc. London, Ser. A 392, 45 (1984).

[45] D. Xiao, M. C. Chang, and Q. Niu, Rev. Mod. Phys. 82, 1959 (2010).

[46] N. Marzari and D. Vanderbilt, Phys. Rev. B 56, 12847 (1997).

[47] M. A. Mead, Rev. Mod. Phys. 64, 51 (1992).

[48] C. Leforestier, R. H. Bisseling, C. Cerjan, M. D. Feit, R. Friesner, A. Guldberg, A. Hammerich, G. Jolicard, W. Karrlein, H. D. Meyer, N. Lipkin, O. Roncero, and R. Kosloff, J. Comput. Phys. 94, 59 (1991).

[49] A. Schleife, E. W. Draeger, Y. Kanai, and A. A. Correa, J. Chem. Phys. 137, 22A546 (2012).

[50] J. K. Tomfohr and O. F. Sankey, Phys. Status Solidi (b) 226, 115 (2001).

[51] M. A. Zeb, J. Kohanoff, D. Sánchez-Portal, A. Arnau, J. I. Juaristi, and E. Artacho, Phys. Rev. Lett. 108, 225504 (2012).

[52] A. A. Correa, J. Kohanoff, E. Artacho, D. Sanchez-Portal, and A. Caro, Phys. Rev. Lett. 108, 213201 (2012).

[53] R. Ullah, F. Corsetti, D. Sanchez-Portal, and E. Artacho, Phys. Rev. B 91, 125203 (2015).

[54] M. C. Payne, M. P. Teter, D. C. Allan, T. A. Arias, and J. D. Joannopoulos, Rev. Mod. Phys. 64, 1045 (1992). 